\title{
The Topological Structure of Polish Groups and Groupoids of Measure Space Transformations
}

\author{
By
}

\author{
Alexandre I. DANILENKO*
}

\begin{abstract}
It is proved that the groupoid of nonsingular partial isomorphisms of a Lebesgue space $(X, \mu)$ is weakly contractible in a "strong" sense: we present a contraction path which preserves invariant the subgroupoid of $\mu$-preserving partial isomorphisms as well as the group of nonsingular transformations of $X$. Moreover, let $\mathscr{R}$ be an ergodic measured discrete equivalence relation on $X$. The full group $[R]$ endowed with the uniform topology is shown to be contractible. For an approximately finite $\mathscr{R}$ of type $I I$ or $I I I_{\lambda}, 0 \leq \lambda<1$, the normalizer $N[\mathscr{R}]$ of $\mathscr{R}$ furnished with the natural Polish topology is established to be homotopically equivalent to the centralizer of the associated Poincare flow. These are the measure theoretical analogues of the resent results of S. Popa and M. Takesaki on the topological structure of the unitary and the automorphism group of a factor.
\end{abstract}

Key words and phrases. Nonsingular transformation, normalizer of full group, contraction path.

The topological properties of automorphism groups of a Lebesgue space $(X$, $\mu$ ) have been studied since 1944 , when P. Halmos [Ha] introduced two metrizable topologies on the group $\operatorname{Aut}_{0}(X, \mu)$ of $\mu$-preserving transformations: the weak $d_{w}$ and the uniform $d_{u}$. He proved that $\left(\operatorname{Aut}_{0}(X, \mu), d_{w}\right)$ is a Polish group. $\mathrm{S}$. Harada [Har] showed that it is simply connected and arcwise connected. His result was later considerably refined by $\mathrm{M}$. Keane $[\mathrm{K}]$ who proved the contractibility of $\operatorname{Aut}_{0}(X, \mu)$ both in $d_{u}$ and $d_{w}$ (see also $[\mathrm{D}, \mathrm{N}]$ ).

A. Ionescu Tulsea [IT] and R. V. Chacon and N. A. Friedman [ChF] extended the weak and the uniform topology to the group $\operatorname{Aut}(X, \mu)$ of nonsingular transformations of $(X, \mu)$ and generalized the results obtained in [Ha]. However, the homotopical properties of this group have not been studied so far.

Communicated by H. Araki, January 30, 1995.

1991 Mathematics Subject Classifications: Primary 55P10, 22A05, 22A22, 28D15; Secondary 46L55.

* Department of Mathematics, Kharkov State University, 4 Freedom sq., Kharkov, 310077, Ukraine.

The work was supported in part by the International Science Foundation Grant No U2B000. 
For a detailed exposition of the productive interplay between ergodic theory and operator algebras we refer to $[\mathrm{M}, \mathrm{C}, \mathrm{S} 2]$. The present work also was stimulated by a paper [PT] being pertained to operator algebras. In particular, given a countable group $\Gamma \subset$ Aut $(X, \mu)$, then one can consider the full group $[\Gamma]$ and its normalizer $N[\Gamma]$ in $\operatorname{Aut}(X, \mu)$ which are the measure theoretical analogues of the unitary group $U(M)$ and the automorphism group Aut $(M)$ of a von Neumann algebra $M$. Both groups, $[\Gamma]$ and $N[\Gamma]$, are Polish: the first with respect to $d_{u}$, the second with respect to some metric $d$ defined by T. Hamachi and M. Osikawa [HO]. Further topological properties of $[\Gamma]$ was discovered by S. I. Bezuglyi and V. Ya. Golodets $[\mathrm{BG} 1]$. They proved that $\left([\Gamma], d_{u}\right)$ is simply connected and arcwise connected (even regardless the countability of $\Gamma$ ). On the other hand it is known that $U(M)$ is contractible for any properly infinite algebra $M$ as well as for a family of type $I I_{1}$ factors [PT]. Moreover, the homotopical structure of Aut $(M)$ is completely described by S. Popa and M. Takesaki [PT] for AFD factors of type $I I$ and $I I I_{\lambda}, 0<\lambda<1$. It seems natural to obtain the similar results for $[\Gamma]$ and $N[\Gamma]$.

In the first section of this paper we study the topological structure of the groupoid Part $(X, \mu)$ of partial isomorphisms of $(X, \mu)$. We show that $\operatorname{Part}(X$, $\mu)$ endowed with the "generalized" weak topology is a Polish groupoid. Furthermore, Part $(X, \mu)$ is contractible in a "strong" sense: we provide a contraction path which preserves invariant both $\operatorname{Aut}(X, \mu)$ and $\operatorname{Aut}_{0}(X, \mu)$. So, $\operatorname{Aut}(X, \mu)$ is also contractible.

In the rest of this paper we prove the statements announced in [Da].

The second section is devoted to the study of homotopical properties of the Polish full group $[\Gamma]$. Note, that $[\Gamma]$ may be identified with the group of inner automorphisms of the $\Gamma$-orbital measured equivalence relation. We first prove that $[\Gamma]$ is contractible if $\Gamma$ is of type $I$ or $I I$. Then using Krieger's discrete decomposition theorems we demonstrate the contractibility of $[\Gamma]$ actually for any ergodic type $I I I$ group $\Gamma$ (only in $I I I_{1}$ case we restrict our consideration to a special class of groups; approximately finite groups are in this class). As an auxiliary result we describe the structure of any ergodic $I I I_{0}$ transformation group (see also [B]). This generalizes the Krieger's theorem for approximately finite groups.

In the final section we investigate the topological structure of $N[\Gamma]$ for an approximately finite ergodic group $\Gamma$. The main results are as follows. If $\Gamma$ is of type $I I$, then $N[\Gamma]$ is contractible. If $\Gamma$ is of type $I I I_{\lambda}, 0<\lambda<1$, then $\pi_{1}(N[\Gamma])$ $=\mathbf{Z}$. For a type $I I I_{0}$ group $\Gamma$ the normalizer $N[\Gamma]$ is homotopically equivalent to the centralizer of the associated Poincare flow endowed with the weak topology. Note that the AFD factors of type $I I I_{0}$ were not considered in [PT].

We prove the above statements in pure measure theoretical setting without use of the modular theory, which was the fundamental tool in [PT]. Moreover, unlike [PT], we do not use the Michael's selection principle, and all of required 
continuous cross-sections are constructed in an explicit way.

Note, that the problem of the contractibility of $N[\Gamma]$ for a type $I I I_{1}$ group $\Gamma$ remains unsolved in spite of the announcement $[\mathrm{Da}]$, since there was an error in the earlier proof.

I am grateful to V. Ya. Golodets for drawing my attention to the paper $[\mathrm{PT}]$; he also suggested to obtain measure theoretical analogues of those results. I thank the participants of the Kharkov Seminar on Ergodic Theory and Operator Algebras for helpful discussion of the work.

\section{§1. The Topological Structure of Aut $(X, \mu)$ and Part $(X, \mu)$}

Let $(X, \mathfrak{B}, \mu)$ be a Lebesgue space; we suppose that $\mu$ is nonatomic and $\sigma$-finite. We fix a map $[0, \mu(X)] \ni s \rightarrow X(s) \in \mathfrak{B}$ with

(i) $\cup_{s} X(s)=X$, and $X(s) \subset X(t)$ if $s<t$;

(ii) $\mu(X(s))=s$ for all $s$.

For a subset $A \in \mathfrak{B}, \mu(A)>0$, consider the function $\phi:[0, \mu(A)] \rightarrow \mathbf{R}_{+}$ given by

$$
\phi(s)=\min \left\{t \in \mathbf{R}_{+} \mid \mu(A \cap X(t))=s\right\} .
$$

Put $A(s)=A \cap X(\phi(s))$. Then $\cup_{s} A(s)=A, A(s) \subset A(t)$ if $s<t$, and $\mu(A(s))=s$ for all $s \in[0, \mu(A)]$. The family $\{A(s)\}_{s}$ will be called the $\mu^{-c o n t i n u o u s ~ d e c o m-~}$ position of $A$. The continuity means that $\mu\left(A_{n}\left(s_{n}\right) \triangle A(s)\right) \rightarrow 0$ if $\mu\left(A_{n} \triangle A\right) \rightarrow 0$ and $s_{n} \rightarrow s$ as $n \rightarrow \infty$.

We let

$\operatorname{Part}(X, \mu)=\{\theta \mid \theta$ is an invertible, nonsingular map from a measurable subset $\operatorname{Dom}(\theta)$ onto a measurable subset $\operatorname{Im}(\theta)\}$,

$\operatorname{Part}_{0}(X, \mu)=\{\theta \in \operatorname{Part}(X, \mu) \mid \theta$ preserves $\mu\}$,

$\operatorname{Aut}(X, \mu)=\{\theta \in \operatorname{Part}(X, \mu) \mid \operatorname{Dom}(\theta)=\operatorname{Im}(\theta)=X\}$,

$\operatorname{Aut}_{0}(X, \mu)=\operatorname{Part}_{0}(X, \mu) \cap \operatorname{Aut}(X, \mu)$.

Note, that we identify any two objects (such as measurable sets, maps, partitions, etc.) which are equal almost everywhere.

We shall suppose below that $\mu(X)=1$. Denote by $\mathscr{B}_{1}(\mathscr{H})$ the unit ball of the algebra of all bounded linear operators of the Hilbert space $\mathscr{H}=L^{2}(X, \mu)$. It is known that $\mathscr{B}_{1}(\mathscr{H})$ endowed with the $*$-strong operator topology is a Polish *-semigroup. The set of all projectors 


$$
\mathscr{P}(\mathscr{H})=\left\{P \in \mathscr{B}_{1}(\mathscr{H}) \mid P=P^{*}, P^{2}=P\right\}
$$

as well as the set of all partial isometries

$$
\operatorname{Iso}(\mathscr{H})=\left\{U \in \mathscr{B}_{1}(\mathrm{H}) \mid U U^{*} \in \mathscr{P}(\mathscr{H})\right\}
$$

are closed subsets of $\mathscr{B}_{1}(\mathscr{H})$. Note that Iso $(\mathscr{H})$ is a groupoid if we set

$$
\operatorname{Iso}^{(2)}(\mathscr{H})=\left\{(U, V) \in \operatorname{Iso}(\mathscr{H}) \times \operatorname{Iso}(\mathscr{H}) \mid U^{*} U=V V^{*}\right\}
$$

and define the multiplication by

$$
\operatorname{Iso}^{(2)}(\mathscr{H}) \ni(U, V) \mapsto U V \in \operatorname{Iso}(\mathscr{H}) .
$$

The unit space of this groupoid is $\mathscr{P}(\mathscr{H})$; the right and the left unit of an element $U \in$ Iso $(\mathscr{H})$ is $U^{*} U$ and $U U^{*}$ respectively; the inversion map is the *-conjugation. Moreover, Iso ${ }^{(2)}(\mathscr{H})$ and $\mathscr{P}(\mathscr{H})$ are closed subsets of Iso $(\mathscr{H}) \times$ Iso $(\mathscr{H})$ and Iso $(\mathscr{H})$ respectively; and all of the groupoid operations are continuous. So, Iso $(\mathscr{H})$ is a Polish groupoid (see $[\mathrm{R}])$. We let

$$
\mathscr{P}_{+}(\mathscr{H})=\left\{P_{A} \mid A \in \mathfrak{B}\right\},
$$

where $P_{A}$ is the projector on the subspace of functions which are vanish outside $A$. The Boolean algebra $\mathscr{P}_{+}(\mathscr{H})$ is a closed subset of $\mathscr{P}(\mathscr{H})$. It is well known that

$$
\left\{P \in \mathscr{P}(\mathscr{H}) \mid P P_{A}=P_{A} P \text { for all } A \in \mathfrak{B}\right\}=\mathscr{P}_{+}(\mathscr{H})
$$

We set

$$
\mathrm{IsO}_{+}(\mathscr{H})=\left\{U \in \mathrm{Iso}(\mathscr{H}) \mid U \mathscr{P}+(\mathscr{H}) U^{*} \subset \mathscr{P}_{+}(\mathscr{H}), U 1 \in \mathscr{H}_{+}\right\}
$$

where $\mathscr{H}_{+}=L_{+}^{2}(X, \mu)$ is the closed cone of almost everywhere nonnegative functions. It is obvious that $\mathrm{IsO}_{+}(\mathscr{H})$ is a closed subgroupoid of Iso $(\mathscr{H})$.

For a partial isomorphism $\theta \in \operatorname{Part}(X, \mu)$ the function

$$
\operatorname{Dom}(\theta) \ni x \mapsto \frac{d \mu \circ \theta}{d \mu}(x) \in \mathbb{R}
$$

is well defined. We extend it to the whole $X$ by setting $\frac{d \mu \circ \theta}{d \mu}(x)=0$ for all $x \notin$ $\operatorname{Dom}(\theta)$. So, the linear bounded operator $U_{\theta}$ of $\mathscr{H}$ given by 


$$
\left(U_{\theta} f\right)(x)=f(\theta x) \sqrt{\frac{d \mu \circ \theta}{d \mu}(x)}, \quad x \in X,
$$

is well defined. It is obvious that $U_{\theta}$ belongs to Iso $(\mathscr{H})$ and $U_{\theta}^{*} U_{\theta}=P_{\operatorname{Dom}(\theta)}, U_{\theta} U_{\theta}^{*}$ $=P_{\operatorname{Im}(\theta)}$. We shall show that the one-to-one groupoid antiisomorphism

$$
\operatorname{Part}(X, \mu) \ni \theta \mapsto U_{\theta} \in \mathrm{Iso}_{+}(\mathscr{H})
$$

is onto. Actually let $U \in \mathrm{IsO}_{+}(\mathscr{H})$ with $U^{*} U=P_{A}$ and $U U^{*}=P_{B}$ for some measurable subsets $A, B \in \mathfrak{B}$. Notice that either $\mu(A)>0$ and $\mu(B)>0$ or $\mu(A)=\mu(B)$ $=0$. Then $U$ induces an isomorphism of the Boolean algebra generated by $(A, \mathfrak{B}$ $\lceil A, \mu\lceil A)$ onto that generated by $(B, \mathfrak{B} \backslash B, \mu\lceil B)$. By the property of Lebesgue space we have that $U=M_{f} U_{\theta}$ for some $\theta \in \operatorname{Part}(X, \mu)$ with $\operatorname{Dom}(\theta)=A$ and $\operatorname{Im} \theta=B$, where $M_{f}$ is the multiplication operator, $f \in L^{\infty}(X, \mu)$, and $|f(x)|=1$ for almost all $x \in A$. Since $U 1 \in \mathscr{H}_{+}, f(x)=1$ almost everywhere on $A$. Hence $U=U_{\theta}$. So we may identify $\operatorname{Part}(X, \mu)$ with $\mathrm{IsO}_{+}(\mathscr{H})$. Since $\mathrm{IsO}_{+}(\mathscr{H})$ is a closed subgroupoid of Iso $(\mathscr{H})$, it follows that $\operatorname{Part}(X, \mu)$ is a Polish groupoid. This topology on $\operatorname{Part}(X, \mu)$ will be called weak and denoted by $d_{w}$ since its restriction to Aut $(X, \mu)$ is the well known weak topology introduced in [Ha, IT]. Note that Aut $(X, \mu)$ corresponds to the stability group of the identity operator (as an element of $\left.\mathrm{IsO}_{+}(\mathscr{H})\right)$. Hence we identify it with

$$
\mathscr{U}_{+}(\mathscr{H})=\left\{U \in \mathscr{U}(\mathscr{H}) \mid U \mathscr{P}_{+}(\mathscr{H}) U^{*}=\mathscr{P}_{+}(\mathscr{H}), U 1 \in \mathscr{H}_{+}\right\}
$$

(both in algebraic and topological aspects), where $\mathcal{U}(\mathscr{H})$ is the unitary group in $\mathscr{H}$.

Proposition 1.1. (i) $d_{w}$ is unaffected if we replace $\mu$ by an equivalent measure;

(ii) $d_{w}$ is induced by the family of pseudometrics:

$$
\begin{aligned}
& p_{-}\left(\theta_{1}, \theta_{2}\right)=\left\|\frac{d \mu \circ \theta_{1}^{-1}}{d \mu}(.)-\frac{d \mu \circ \theta_{2}^{-1}}{d \mu}(.)\right\|_{1} \quad \text { and } \\
& p_{A}\left(\theta_{1}, \theta_{2}\right)=\mu\left(\theta_{1}\left(A \cap \operatorname{Dom}\left(\theta_{1}\right)\right) \triangle \theta_{2}\left(A \cap \operatorname{Dom}\left(\theta_{2}\right)\right)\right), \quad A \in \mathfrak{B},
\end{aligned}
$$

for $\theta_{1}, \theta_{2} \in \operatorname{Part}(X, \mu)$.

Proof. The corresponding statements for Aut $(X, \mu)$ were proved in [Ha, IT, HO]. In our case one should apply a similar argument.

It is known that $\operatorname{Aut}_{0}(X, \mu)$ is contractible $[\mathrm{K}]$. We shall prove the following 
Theorem 1.2. The groupoid $\operatorname{Part}_{0}(X, \mu)$ furnished with the weak topology is contractible in such a way that there exists a continuous map

$$
\sigma:[0,1] \times \operatorname{Part}_{0}(X, \mu) \ni(\mathrm{t}, \theta) \mapsto \sigma_{t}(\theta) \in \operatorname{Part}_{0}(X, \mu)
$$

with the properties

(i) $\sigma_{0}(\theta)=\theta, \quad \sigma_{1}(\theta)=\left.\mathrm{id}\right|_{X}$

(ii) $\sigma_{t}\left(\operatorname{Aut}_{0}(X, \mu)\right) \subset \operatorname{Aut}_{0}(X, \mu) \quad$ for each $t \in[0,1]$.

Proof. For a partial isomorphism $\theta \in \operatorname{Part}_{0}(X, \mu)$ we set

$$
\begin{gathered}
Y=\operatorname{Dom}(\theta), A=Y \cap \operatorname{Im}(\theta), B=\{x \in Y \mid \theta x \in A\}, \\
D=Y-B, C=Y-A, E=X-Y .
\end{gathered}
$$

We also denote $u=\mu(C)=\mu(D), v=\mu(Y)$. Let $\zeta$ be the (unique) partial isomorphism which is determined by the conditions: $\operatorname{Dom}(\zeta)=D, \operatorname{Im}(\zeta)=C$, and $\zeta D(t)=C(t)$ for every $t \in[0, u]$. Remind that $\{C(t)\}_{t},\{D(t)\}_{t}$ are the $\mu$-continuous decompositions of $C$ and $D$ respectively. Then we define a partial isomorphism $\tilde{\theta}$ with $\operatorname{Dom}(\tilde{\theta})=\operatorname{Im}(\tilde{\theta})=Y$ by

$$
\tilde{\theta} x= \begin{cases}\theta x, & \text { for } x \in B \\ \zeta x, & \text { for } x \in D\end{cases}
$$

It is straightforward that the map

$$
\operatorname{Part}_{0}(X, \mu) \ni \theta \mapsto \tilde{\theta} \in \operatorname{Part}_{0}(X, \mu)
$$

is continuous. Since $\tilde{\theta}$ is a measure preserving automorphism of the finite measure space $(Y, \mu \uparrow Y)$, it generates for each $t \in[0, v]$ the induced automorphism $\tilde{\theta}_{t}$ of the space $(Y(v-t), \mu \uparrow Y(v-t))$. Now we set

$$
\operatorname{Dom}\left(\sigma_{t}(\theta)\right)= \begin{cases}Y, & \text { for } t \in[0, v] \\ Y \cup E(t-v), & \text { for } t \in(v, 1]\end{cases}
$$

and define

$$
\begin{aligned}
& \sigma_{t}(\theta) x=\left\{\begin{array}{ll}
\tilde{\theta}_{t} x, & \text { for } x \in Y(v-t) \\
x, & \text { for } x \in Y-Y(v-t)
\end{array} \quad \text { if } t \in[0, v] ;\right. \\
& \sigma_{t}(\theta) x=x \quad \text { for all } x \in \operatorname{Dom}\left(\sigma_{t}(\theta)\right)
\end{aligned}
$$


It is routine to check that $\sigma$ is the desired map.

Without loss in generality, we may assume that $X$ is the unit interval $[0,1]$ and $\mu$ the Lebesgue measure. We shall use the notation

$$
\begin{aligned}
& \mathcal{M}=\left\{f \in L^{1}(X, \mu) \mid\|f\|_{1} \leq 1, f(x) \geq 0 \text { for a.a. } x\right\} \\
& \mathcal{N}=\left\{f \in L^{1}(X, \mu) \mid\|f\|_{1}=1, f(x)>0 \text { for a.a. } x\right\}
\end{aligned}
$$

For a function $f \in \mathcal{M}$ we set $\operatorname{Supp}(f)=\{x \in X \mid f(x)>0\}$ and $s(f) x=\int_{0}^{x} f(t) d \mu(t)$. It is easy to see that $s(f)$ is a partial isomorphism with $\operatorname{Dom}(s(f))=\operatorname{Supp}(f)$ and $\operatorname{Im}(s(f))=\left[0,\|f\|_{1}\right]$ with

$$
\frac{d \mu \circ_{s}(f)}{d \mu}=f, \quad \frac{d \mu \circ_{s}(f)^{-1}}{d \mu}(x)=1 / f\left(s(f)^{-1} x\right)
$$

for all $x \in \operatorname{Im}(s(f))=\operatorname{Dom}\left(s(f)^{-1}\right)$.

We define now a metric $m$ on $\mathcal{M}$ by

$$
m\left(f_{1}, f_{2}\right)=\left\|f_{1}-f_{2}\right\|_{1}+\left\|\frac{d \mu \circ s\left(f_{1}\right)^{-1}}{d \mu}-\frac{d \mu \circ s\left(f_{2}\right)^{-1}}{d \mu}\right\|_{1}
$$

for $f_{1}, f_{2} \in \mathcal{M}$. Then $\mathcal{M}$ endowed with $m$ is a Polish space and $\mathcal{N}$ is its closed subset. We put

$$
\mathcal{M} \star \operatorname{Part}_{0}(X, \mu)=\left\{(f, \zeta) \in \mathcal{M} \times \operatorname{Part}_{0}(X, \mu) \mid \operatorname{Im}(\zeta)=\left[0,\|f\|_{1}\right]\right\} .
$$

Then $\mathcal{M} \operatorname{Part}_{0}(X, \mu)$ is a closed subset of $\mathcal{M} \times \operatorname{Part}_{0}(X, \mu)$.

Theorem 1.3. (i) There is a homeomorphism from $\operatorname{Part}(X, \mu)$ onto $M *$ $\operatorname{Part}_{0}(X, \mu)$ which takes Aut $(X, \mu)$ (respectively $\left.\operatorname{Part}_{0}(X, \mu)\right)$ onto $\mathcal{N} \times \operatorname{Aut}_{0}(X, \mu)$ (respectively $\left.\{1\} \times \operatorname{Part}_{0}(X, \mu)\right)$;

(ii) $\operatorname{Part}(X, \mu)$ is contractible in such a way that there exists a continuous map

$$
\hat{\sigma}:[0,1] \times \operatorname{Part}(X, \mu) \ni(t, \theta) \mapsto \hat{\sigma}_{t}(\theta) \in \operatorname{Part}(X, \mu)
$$

with the properties: $\hat{\sigma}_{0}(\theta)=\theta, \quad \hat{\sigma}_{1}(\theta)=\left.\mathrm{id}\right|_{X}$, and

$$
\begin{aligned}
\hat{\sigma}_{t}(\operatorname{Aut}(X, \mu)) & \subset \operatorname{Aut}(X, \mu) \\
\hat{\sigma}_{t}\left(\operatorname{Part}_{0}(X, \mu)\right) & \subset \operatorname{Part}_{0}(X, \mu)
\end{aligned} \quad \text { for each } t \in[0,1] .
$$

Proof. (i) Consider two maps $\pi, s$ given by 


$$
\begin{aligned}
& \pi: \operatorname{Part}_{0}(X, \mu) \ni \theta \mapsto \pi(\theta)=\frac{d \mu \circ \theta}{d \mu} \in \mathcal{M}, \\
& s: \mathcal{M} f_{f} \mapsto s(f) \in \operatorname{Part}_{0}(X, \mu) .
\end{aligned}
$$

A routine verification shows that both of them are continuous. Moreover, the following properties are satisfied:

$$
\begin{aligned}
\pi \circ s=\mathrm{id}, & \pi(\zeta \theta)=\pi(\theta) \\
s(\pi(\theta)) \theta^{-1} \in \operatorname{Part}_{0}(X, \mu), & \operatorname{Im}\left(s(\pi(\theta)) \theta^{-1}\right)=\left[0,\|\pi(\theta)\|_{1}\right]
\end{aligned}
$$

for all $\theta \in \operatorname{Part}(X, \mu), \zeta \in \operatorname{Part}_{0}(X, \mu)$ with $\operatorname{Im}(\theta)=\operatorname{Dom}(\zeta)$. So, the map

$$
\operatorname{Part}_{0}(X, \mu) \ni \theta \mapsto\left(\pi(\theta), s(\pi(\theta)) \theta^{-1}\right) \in \mathcal{M}
$$

is a homeomorphism as desired.

(ii) The indicator of a set $B \in \mathfrak{B}$ will be denoted by $I_{B}$. Note, that the space $\mathcal{M}$ endowed with $m$ is contractible. Moreover, it is not difficult to construct a continuous map

$$
\tilde{\sigma}:[0,1] \times \mathcal{M} \ni(t, f) \mapsto \tilde{\sigma}_{t}(f) \in \mathcal{M}
$$

with the following properties: $\quad \tilde{\sigma}_{0}(f)=f, \tilde{\sigma}_{1}(f)=I_{X}$,

$$
\begin{aligned}
\left\|\tilde{\sigma}_{t}(f)\right\|_{1}=\|f\|_{1} & \text { for all } t \in\left[0,\|f\|_{1}\right], \\
\tilde{\sigma}_{t}(f)=I_{[0, t]} & \text { for all } t \in\left[\|f\|_{1}, 1\right], \\
\tilde{\sigma}_{t}(\mathcal{N}) \subset \mathcal{N} & \text { for all } t \in[0,1] .
\end{aligned}
$$

Then for each $(f, \zeta) \in \mathcal{M} \star \operatorname{Part}_{0}(X, \mu)=\operatorname{Part}(X, \mu)$ and $\mathrm{t} \in[0,1]$ we set

$$
\hat{\sigma}_{t}(f, \zeta)=\left(\tilde{\sigma}_{t}(f), \sigma_{t}\left(\zeta^{-1}\right)^{-1}\right)
$$

where $\left\{\sigma_{t}\right\}_{t}$ is the contraction path determined in Theorem 1.2. It is easy to see that the map $\hat{\sigma}$ is as desired.

\section{§2. Contractibility of Full Groups and Groupoids}

2.1. The uniform topology on $\operatorname{Part}(X, \mu)$ is generated by the metric $d_{u}$ : $d_{u}\left(\theta_{1}, \theta_{2}\right)=\mu\left(\left\{x \in \operatorname{Dom}\left(\theta_{1}\right) \cap \operatorname{Dom}\left(\theta_{2}\right) \mid \theta_{1} \neq \neq \theta_{2} x\right\}\right)$ 


$$
\begin{aligned}
& +\mu\left(\left\{x \in \operatorname{Im}\left(\theta_{1}\right) \cap \operatorname{Im}\left(\theta_{2}\right) \mid \theta_{1}^{-1} x \neq \theta_{2}^{-1} x\right\}\right) \\
& +\mu\left(\operatorname{Dom}\left(\theta_{1}\right) \triangle \operatorname{Dom}\left(\theta_{2}\right)\right)+\mu\left(\operatorname{Im}\left(\theta_{1}\right) \triangle \operatorname{Im}\left(\theta_{2}\right)\right),
\end{aligned}
$$

for $\theta_{1}, \theta_{2} \in \operatorname{Part}(X, \mu)$

It induces the well known uniform topology on the closed subset $\operatorname{Aut}(X, \mu)$ $\subset \operatorname{Part}(X, \mu)$. Like it was done in the case of the group Aut $(X, \mu)$ [IT, HO], one can prove that $\operatorname{Part}(X, \mu)$ is a complete, nonseparable, topological groupoid and that the uniform topology is stronger than the weak one.

For a countable subgroup $\Gamma \subset \operatorname{Aut}(X, \mu)$ we set

$$
\begin{aligned}
{[\Gamma]_{*} } & =\{\theta \in \operatorname{Part}(X, \mu) \mid \theta x \in \Gamma x \quad \text { for a.e. } x \in \operatorname{Dom}(\theta)\}, \\
{[\Gamma] } & =[\Gamma]_{*} \cap \operatorname{Aut}(X, \mu) .
\end{aligned}
$$

Then $[\Gamma]$ (respectively $[\Gamma]_{*}$ ) is said to be the full group (respectively the full groupoid) generated by $\Gamma$.

The normalizer of $[\Gamma]$ in $\operatorname{Aut}(X, \mu)$ will be denoted by $N[\Gamma]$, i.e.

$$
N[\Gamma]=\left\{\theta \in \operatorname{Aut}(X, \mu) \mid \theta[\Gamma] \theta^{-1}=[\Gamma]\right\}
$$

It is known that $[\Gamma]$ is a Polish group with respect to the uniform topology [HO]. In a similar way, $[\Gamma]_{*}$ endowed with $d_{u}$ is a Polish groupiod.

Note, that the uniform and the weak topologies are coincide on $[\Gamma]$ if $\Gamma$ is finite or, more generally, is type $I$.

It is easy to see that $[\Gamma]_{*}$ is contractible with respect to the uniform topology. Our purpose now is to study the homotopical properties of the full group $[\Gamma]$ endowed with $d_{u}$.

Proposition 2.1. Let $\Gamma$ be a group of a finite type, i.e. there exist a $\Gamma$-invariant, finite measure equivalent to $\mu$. Then $[\Gamma]$ is contractible.

Proof. Consider the contraction path for $\left(\operatorname{Aut}_{0}(X, \mu), d_{w}\right)$ constructed in [K] (see also Theorem 1.3). It uses the induced transformations. Then its restriction to $[\Gamma]$ is the contraction path for $[\Gamma]$ endowed with the uniform topology.

Note that if $\Gamma$ is of infinite type, then the method of induced transformations is not applicable, since an arbitrary automorphism $\gamma \in \Gamma$ needs not to be conservative.

2.2. In this subsection we consider the case where $\Gamma$ is of semifinite type. The main result is as follows. 
Theorem 2.2. Let there exist a $\sigma$-finite, $\Gamma$-invariant, $\mu$-equivalent measure ע. Then $[\Gamma]$ is contractible.

Proof. We divide the proof of this proposition into some steps.

(s-1) Suppose first that $\Gamma$ is ergodic and type $I I_{\infty}$. We fix a $\nu$-continuous decomposition of $X=\cup_{s_{\geq 0}} X(s)$ with $\nu(X(s))=s$. Denote by $\mathscr{P}_{1}$ the family of countable ordered partitions $\eta=\left\{\eta_{n}\right\}_{n=1}^{\infty}$ of $X$ with $\nu\left(\eta_{n}\right)=1$ for all $n \in \mathbb{N}$. It is easy to see that $\mathscr{P}_{1}$ is a Polish space with respect to the metric $r$ given by

$$
r(\vartheta, \eta)=\sum_{n} \mu\left(\vartheta_{n} \Delta \eta_{n}\right), \quad \vartheta, \eta \in \mathscr{P}_{1}
$$

Moreover, the topology on $\mathscr{P}_{1}$ is uneffected if we replace $\mu$ by an equivalent measure. We now fix a partition $\eta \in \mathscr{P}_{1}$ and set

$$
[\Gamma]_{\eta}=\left\{\gamma \in[\Gamma] \mid \gamma \eta_{n}=\eta_{n} \quad \text { for all } n \in \mathbb{N}\right\}
$$

It is obvious that $[\Gamma]_{\eta}$ is homeomorphic to the Cartesian product $\prod_{n=1}^{\infty}[\Gamma]_{\eta_{n}}$, where $[\Gamma]_{\eta_{n}}$ is the induced (to the subset $\eta_{n}$ ) full group, $n \in \mathbb{N}$ [HO]. It is known that there exists an ergodic type $I I_{1}$ transformation group $\Gamma_{n}$ on the space $\left(\eta_{n}, \mu\left\lceil\eta_{n}\right)\right.$ with $\left[\Gamma_{n}\right]=[\Gamma]_{\eta_{n}}, n \in \mathbb{N}$. Then in view of Proposition $2.1,[\Gamma]_{n}$ is contractible closed subgroup of $[\Gamma]$. Consider the map

$$
\pi:[\Gamma] \ni \gamma \mapsto \pi(\gamma)=\left\{\pi_{n}(\gamma)\right\}_{n=1}^{\infty} \in \mathscr{P}_{1},
$$

where $\pi_{n}(\gamma)=\gamma \eta_{n}$ for all $n \in \mathbb{N}$. It is continuous and constant on the left $[\Gamma]_{\eta}$ -cosets. We need two auxiliary lemmas.

Lemma 2.3. $\mathscr{P}_{1}$ is contractible.

Proof. We set

$$
\mathscr{P}_{1}(k)=\left\{\vartheta \in \mathscr{P}_{1} \mid \vartheta_{n}=\eta_{n} \quad \text { for } n=1, \ldots k\right\}
$$

Then we have a decreasing sequence of closed subsets

$$
\mathscr{P}_{1}(1) \supset \mathscr{P}_{1}(2) \supset \ldots, \quad \text { with } \bigcap_{k=1}^{\infty} \mathscr{P}_{1}(k)=\{\eta\}
$$

For a partition $\vartheta \in \mathscr{P}_{1}$ we set $B=\vartheta_{1}-\eta_{1}, A_{n}=\left(\eta_{1}-\vartheta_{1}\right) \cap \vartheta_{n}, s_{1}=0, s_{n}=\nu\left(A_{2}\right)+$ $\cdots+\nu\left(A_{n}\right), B_{n}=B\left(s_{n}\right)-B\left(s_{n-1}\right)$, where $n=2,3, \ldots$. Now for each real $t \in[0$, $\nu(B)]$ there is an integer $m$ with $s_{m} \leq t<s_{m-1}$. We set 


$$
\delta_{t}^{(1)}(\vartheta)_{n}= \begin{cases}\left(\vartheta_{1} \cap \eta_{1}\right) \cup{ }_{i=2}^{m} A_{i} \cup A_{m+1}\left(t-s_{m}\right) \cup(B-B(t)), & \text { for } n=1 \\ \left(\vartheta_{n}-A_{n}\right) \cup B_{n}, & \text { for } n=2, \ldots, m \\ \left(\vartheta_{n}-A_{n}\left(t-s_{m}\right)\right) \cup B_{n}\left(t-s_{m}\right), & \text { for } n=m+1 \\ \vartheta_{n} & \text { for } n>m+1\end{cases}
$$

Then the partition $\delta_{t}^{(1)}(\vartheta)=\left\{\delta_{t}^{(1)}(\vartheta)_{n}\right\}_{n} \in \mathscr{P}_{1}$ for $t \in[0, \nu(B))$. Since the map $\mathscr{P}_{1} \ni \vartheta \mapsto \nu\left(\vartheta_{1}-\eta_{1}\right) \in \mathbb{R}$ is continuous, the map

$$
\mathscr{P}_{1} \times[0,1) \ni(\vartheta, s) \mapsto \sigma_{s}^{(1)}(\vartheta) \stackrel{\text { def }}{=} \delta_{s \nu\left(\vartheta_{1}-\eta_{1}\right)}^{(\vartheta)}(\vartheta) \mathscr{P}_{1}
$$

is also continuous. Moreover, $\sigma_{0}^{(1)}(\vartheta)=\vartheta, \sigma_{1-0}^{(1)}(\vartheta) \in \mathscr{P}_{1}(1)$.

In a similar way, for each $k \in \mathbb{N}$ we have a continuous map

$$
\mathscr{P}_{1} \times[0,1) \ni(\vartheta, s) \mapsto \sigma_{s}^{(k+1)}(\vartheta) \in \mathscr{P}_{1}(k)
$$

with $\sigma_{0}^{(k+1)}(\vartheta)=\vartheta$ and $\sigma_{1-0}^{(k+1)}(\vartheta) \in \mathscr{P}_{1}(k+1)$. Now for a positive $s$ we choose $n \in$ $\mathbb{N}$ and $t \in[0,1)$ with $n-1 \leq_{s}<n$ and $s=n-1+t$ and set $\sigma_{s}(\vartheta)=\sigma_{t}^{(n)} \circ \sigma_{1-0}^{(n-1)} \circ$ $\cdots \circ \sigma_{1-0}^{(1)}(\vartheta), \vartheta \in \mathscr{P}_{1}$. It is a routine to check that the map

$$
\sigma: \mathscr{P}_{1} \times \mathbb{R}_{+} \ni(\vartheta, s) \mapsto \sigma_{s}(\vartheta) \in \mathscr{P}_{1}
$$

is continuous and $\sigma_{0}(\vartheta)=\vartheta, \sigma_{+\infty}(\vartheta)=\eta$ for every $\vartheta \in \mathscr{P}_{1}$.

Lemma 2.4. Under the above assumptions ( $\Gamma$ is ergodic), for any two measurable subsets $A, B \in \mathfrak{B}$ with $\nu(A)=\nu(B)>0$ one can choose a partial isomorphism $\gamma(A, B) \in[\Gamma]_{*}$ in such a way that

(i) $\operatorname{Dom}(\gamma(A, B))=A, \operatorname{Im}(\gamma(A, B))=B$,

(ii) if $\left\{A_{n}\right\}_{n=1}^{\infty}$ and $\left\{B_{n}\right\}_{n=1}^{\infty}$ are the two sequences of measurable subsets with $\nu\left(A_{n} \triangle A\right) \rightarrow 0$ and $\nu\left(B_{n} \triangle B\right) \rightarrow 0$, then $\gamma\left(A_{n}, B_{n}\right) \rightarrow \gamma(A, B)$ in the uniform topology as $n \rightarrow \infty$.

Proof. We use a slight modification of the Kantor-Bernstein map. Let $\Gamma=$ $\left\{\gamma_{n}\right\}_{n=1}^{\infty}, \gamma_{1}=$ id. We put $A_{1}=A \cup \gamma_{1}^{-1} B, B_{1}=\gamma_{1} A_{1}, C_{1}=A-A_{1}, D_{1}=B-B_{1}$. Then $\nu\left(C_{1}\right)=\nu\left(D_{1}\right)$. We continue $A_{2}=C_{1} \cup \gamma_{2}^{-1} D_{1}, B_{2}=\gamma_{2} A_{2}, C_{2}=C_{1}-A_{2}, D_{2}=D_{1}-B_{2}$ and so on. Since $\Gamma$ is ergodic, $\cap_{n=1}^{\infty} A_{n}=A$ and $\bigcap_{n=1}^{\infty} B_{n}=B$. Now we set

$$
\gamma(A, B) x=\gamma_{n} x \quad \text { for all } x \in A_{n}, n \in \mathbb{N}
$$

It is a routine to check that the map $\gamma(A, B)$ is as desired. 
Continue the proof of Theorem 2.2. For every partition $\vartheta \in \mathscr{P}_{1}$ using Lemma 2.4 we set

$$
\tau(\vartheta) x=\gamma\left(\vartheta_{n}, \eta_{n}\right) x \quad \text { for } x \in \vartheta_{n}, n \in \mathbf{N}
$$

It is easy to see that the map

$$
\tau: \mathscr{P}_{1} \ni \vartheta \mapsto \tau(\vartheta) \in[\Gamma]
$$

is continuous and $\pi \circ \tau=\mathrm{id}$. So, $\tau$ is a cross-section of the projection map $\pi$. It follows that $[\Gamma]$ is homeomorphic to the product $[\Gamma]_{\eta} \times \mathscr{P}_{1}$ (as well as $\mathscr{P}_{1}$ to the quotient space $[\Gamma] /[\Gamma]_{\eta}$ ). In as much as both multiples are contractible, so is $[\Gamma]$.

(s-2) Now suppose that the dynamical system $(X, \mu, \Gamma)$ is of the following form. There are two Lebesgue spaces $\left(X, \mathfrak{B}_{Y}, \lambda\right)$ and $\left(Z, \mathfrak{B}_{Z}, \kappa\right)$ with $\kappa(Z)=1$, $X=Z \times Y, \mathfrak{B}=\mathfrak{B}_{Y} \times \mathfrak{B}_{Z}$, and $\mu$ is equivalent to $\kappa \times \lambda$. For each $\gamma \in \Gamma$ there is a measurable field $Z \ni z \rightarrow \gamma_{z} \in \operatorname{Aut}_{0}(Y, \lambda)$ of $\lambda$-preserving automorphisms [Kr2, HO] such that $\gamma(z, y)=\left(z, \gamma_{z} y\right)$ for a.e. $(z, y) \in X$. The transformation group $\Gamma_{z}$ $=\bigcup_{r \in \Gamma} \gamma_{z}$ is ergodic and type $I I_{\infty}$ for $\kappa$-a.e. $z \in Z$.

By $\mathscr{P}_{1}$ we denote the family of countable partitions $\varpi=\left\{\varpi_{n}\right\}_{n=1}^{\infty}$ of $Y$ with $\lambda\left(\varpi_{n}\right)=1, n \in \mathbf{N}$, and by $r$ the corresponding Polish metric on it (see $\left.(s-1)\right)$.

For each countable partition $\varpi$ of $X$ and $z \in Z$ we consider the partition $\varpi[z]=\left\{\varpi_{n}[z]\right\}_{n}$ of $Y$, where $y \in \varpi_{n}[z] \Longleftrightarrow(z, y) \in \varpi_{n}$. Then $Z \ni z \mapsto \varpi[z]$ is a measurable field of $Y$-partitions. Otherwise, each measurable field of $Y$-partitions generates some $X$-partition and the correspondence $\varpi \Longleftrightarrow(Z \ni z \mapsto$ $\varpi[z])$ is one-to-one. We let

$$
\widetilde{\mathscr{P}}_{1}=\left\{\varpi \mid \varpi \text { is a countable partition of } X \text { with } \varpi[z] \in \mathscr{P}_{1} \text { for a.e. } z \in Z\right\} \text {. }
$$

Then $\widetilde{\mathscr{P}}_{1}$ is a Polish space with respect to the metric $\widetilde{r}$, given by

$$
\widetilde{r}(\varpi, \eta)=\int_{z} r(\varpi[z], \eta[z]) d \kappa(z), \quad \varpi, \eta \in \widetilde{\mathscr{P}}_{1}
$$

We fix a partition $\eta \in \widetilde{\mathscr{P}}_{1}$ and follow (s-1) in setting up

$$
\Gamma_{n}=\left\{\gamma \in[\Gamma] \mid \gamma \eta_{n}=\eta_{n} \quad \text { for all } n \in \mathbf{N}\right\}
$$

Then in view of Proposition 2.1, the closed subgroup $[\Gamma]_{\eta} \subset[\Gamma]$ is contractible (see $(s-1))$.

Let $\sigma=\left(\sigma_{t}\right)_{t \geq 0}$ be a contraction path for $\mathscr{P}_{1}$ (see Lemma 2.3). We define a map 


$$
\tilde{\sigma}: \widetilde{\mathscr{P}}_{1} \times[0,+\infty) \ni(\varpi, s) \mapsto \widetilde{\sigma}_{s}(\varpi) \in \widetilde{\mathscr{P}}_{1}
$$

by $\left(\tilde{\sigma}_{s}(\varpi)\right)[z]=\sigma_{s}(\varpi[z]), z \in Z$. It is a routine to check that $\tilde{\sigma}$ is well defined. Since $\tilde{\sigma}$ is continuous and $\tilde{\sigma}_{0}(\varpi)=\varpi, \quad \tilde{\sigma}_{\infty}(\varpi)=\eta$, we have that $\widetilde{\mathscr{P}}_{1}$ is contractible. So, just as in $\left(s^{-1}\right)$, to prove the contractibility of $[\Gamma]$, it suffices to present a homeomorphism of $[\Gamma]$ onto $[\Gamma]_{\eta} \times \widetilde{\mathscr{P}}_{1}$. This results from the following analogue of Lemma 2.4 .

Lemma 2.5. Under the above assumptions ( $\mathrm{s}-2)$, let us be given any two measurable subsets $A, B \subset$ with

$$
\lambda(\{y \mid(z, y) \in A\})=\lambda(y \mid(z, y) \in B) \quad \text { for a.e. } z \in Z \text {. }
$$

Then one can choose a partial isomorphism $\gamma(A, B) \in[\Gamma] *$ in such a way that properties (i) and (ii) of Lemma 2.4 are satisfied.

This statement is proved in a similar way as Lemma 2.4. but with reference to the following Krieger's lemma (see [HO] for the proof).

Lemma 2.6. Under the above assumptions for any two measurable subsets $A$, $B \subset X$ satisfying (2-1) there is a partial isomorphism $\theta \in[\Gamma] *$ with $\operatorname{Dom}(\theta)=A$ and $\operatorname{Im}(\theta)=B$.

$(\mathrm{s}-3)$ Now consider the general case. Then $X$ can be decomposed into a union of three pairwise disjoint $\Gamma$-invariant subsets $X=A \cup B \cup C$ in such a way that $[\Gamma]_{A}$ is of finite type, $[\Gamma]_{B}$ is of type $I_{\infty}$, and $[\Gamma]_{C}$ is the full group described in (s-2). Since $[\Gamma]=[\Gamma]_{A} \times[\Gamma]_{B} \times[\Gamma]_{C}$ and each multiple is contractible, $[\Gamma]$ is also contractible. So, the theorem is completely proved.

2.3. In this subsection we consider the case of type $I I I_{\lambda}, 0<\lambda \leq 1$, full group $[\Gamma]$. Remind that a measure $\nu$ on $X$ is called strictly $\Gamma$-admissible $[\mathrm{Krl}$, $\mathrm{HO}]$ if

(i) there is a countable subgroup $R_{d} \subset \mathbb{R}_{+}^{*}$ with $\frac{d \nu \circ \gamma}{d \nu}(x) \in R_{d}$ for all $\gamma \in \Gamma$ at a.e. $x$; and

(ii) there is an ergodic countable subgroup $\Delta \subset[\Gamma]$ with $[\Delta]=[\Gamma] \cap$ $\operatorname{Aut}_{0}(X, \mu)$.

Theorem 2.7. Let $\Gamma$ be an ergodic transformation group of type $I I I_{\lambda}, 0<\lambda<1$. Then $[\Gamma]$ furnished with the uniform topology is contractible.

Proof. Let $\nu$ be a $\mu$-equivalent strictly $\Gamma$-admissible measure on $X$ (existence of such a measure for a type $I I I_{\lambda}$ group was established in $[\mathrm{Krl}$, 
HO]). Since $R_{d}=\lambda^{\mathrm{Z}}$, there are a subset $A \subset X, \mu(A)>0$, and an automorphism $\gamma_{0}$ $\in \Gamma$ with $\frac{d \nu \circ \gamma_{0}}{d \nu}(x)=\lambda$ for all $x \in A$. Without loss in generality we assume that $\nu$ is infinite. Then there exist two sequences $\left\{\gamma_{n}\right\}_{n=1}^{\infty}$ and $\left\{\delta_{n}\right\}_{n=1}^{\infty}$ of automorphisms from $[\Delta]$ such that $\left\{\gamma_{n} A\right\}_{n=1}^{\infty}$ and $\left\{\delta_{n} \gamma_{0} A\right\}_{n=1}^{\infty}$ are partitions of $X$. We set

$$
\theta_{\lambda} x=\delta_{n} \gamma_{0} \gamma_{n}^{-1} x \quad \text { for all } \quad x \in \gamma_{n} A, n \in \mathbb{N} \text {. }
$$

Then $\theta_{n}$ is an automorphism of $(X, \mu), \theta \in \Gamma, \frac{d \nu \circ \theta_{\lambda}}{d \nu}(x)=\lambda$ for a.e. $x$, $\theta_{\lambda}[\Delta] \theta_{\lambda}^{-1}=[\Delta]$, and $[\Gamma]$ is generated by $\Delta$ and $\theta_{\lambda}$.

We may assume that $X=[0, \infty), \nu$ is a Lebesgue measure on $X$, and $d \mu(x)$ $=\exp (-x) d \nu(x)$ for all $x \in X$.

By $\mathscr{P}_{\lambda}$ we denote the family of ordered partitions $\varpi=\left\{\varpi_{n}\right\}_{n \in \mathbf{Z}}$ of $X$ with $\sum_{n \in \mathbf{Z}} \lambda^{n} \nu\left(\varpi_{n}\right)=+\infty$. We admit that some elements of the partitions can be $\nu$-neglectible.

For every automorphism $\gamma \in[\Gamma]$ we define a partition $\pi(\gamma)=\left\{\pi_{n}(\gamma)\right\}_{n} \in \mathscr{P}_{\lambda}$ by

$$
x \in \pi_{n}(\gamma) \Longleftrightarrow \gamma x=\delta_{n} \theta_{\lambda}^{n} x \quad \text { for some } \delta_{n} \in[\Delta], n \in \mathbb{Z}
$$

Then $\pi(\gamma) \in \mathscr{P}_{\lambda}$ and the map

$$
\pi:[\Gamma] \ni \gamma \mapsto \pi(\gamma) \in \mathscr{P}_{\lambda}
$$

is constant on the right $[\Delta]$-cosets. Since $\Delta$ is ergodic, $\mathscr{P}_{\lambda}$ is algebraically isomorphic to the quotient space $[\Delta] \backslash[\Gamma]$. Now we find an explicit expression for the quotient metric $r$ on $\mathscr{P}_{\lambda}$ given by

$$
r\left(\pi\left(\gamma_{1}\right), \pi\left(\gamma_{2}\right)\right)=\inf _{\delta 1, \delta 2 \in[\Delta]} d_{u}\left(\delta_{1} \gamma_{1}, \delta_{2} \gamma_{2}\right), \quad \gamma_{1}, \gamma_{2} \in[\Gamma]
$$

To this end for every pair $\gamma_{1}, \gamma_{2} \in[\Gamma]$ we set

$$
M\left(\gamma_{1}, \gamma_{2}\right)=\left\{x \in X \mid \gamma_{1} x=\gamma_{2} x\right\}, \quad N\left(\gamma_{1}, \gamma_{2}\right)=\underset{n \in \mathbf{Z}}{\bigcup} \pi_{n}\left(\gamma_{1}\right) \cap \pi_{n}\left(\gamma_{2}\right)
$$

Then $M\left(\gamma_{1}, \gamma_{2}\right) \subset N\left(\gamma_{1}, \gamma_{2}\right)$ and $d_{u}\left(\gamma_{1}, \gamma_{2}\right)=2-\mu\left(M\left(\gamma_{1}, \gamma_{2}\right)\right)-\mu\left(\gamma_{1} M\left(\gamma_{1}, \gamma_{2}\right)\right)$. Since $\nu\left(\gamma_{1} N\left(\gamma_{1}, \gamma_{2}\right)\right)=\nu\left(\gamma_{2} N\left(\gamma_{1}, \gamma_{2}\right)\right)$ and $\nu$ is $\Delta$-invariant, there are two transformations $\delta_{1}, \delta_{2} \in[\Delta]$ with

$$
\nu\left(X-\delta_{1} \gamma_{1} N\left(\gamma_{1}, \gamma_{2}\right)\right)=\nu\left(X-\delta_{2} \gamma_{2} N\left(\gamma_{1}, \gamma_{2}\right)\right)=\infty
$$


Then there exists a transformation $\delta_{3} \in[\Delta]$ such that $M\left(\delta_{3} \delta_{1} \gamma_{1}, \delta_{2} \gamma_{2}\right)=N\left(\gamma_{1}, \gamma_{2}\right)$. Since $M\left(\delta \gamma_{1}, \delta \gamma_{2}\right)=M\left(\gamma_{1}, \gamma_{2}\right)$ for each $\delta \in[\Delta]$, we have

$$
\begin{aligned}
r\left(\pi\left(\gamma_{1}, \gamma_{2}\right)\right) & =2-\sup _{\delta_{4}, \delta_{5} \in[\Delta]}\left(\mu\left(M\left(\delta_{4} \gamma_{1}, \delta_{5} \gamma_{2}\right)\right)+\mu\left(\delta_{4} \gamma_{1} M\left(\delta_{4} \gamma_{1}, \delta_{5} \gamma_{2}\right)\right)\right) \\
& =2-\mu\left(N\left(\gamma_{1}, \gamma_{2}\right)\right)-\sup _{\delta 4 \in[\Delta]} \mu\left(\delta_{4} \gamma_{1} N\left(\gamma_{1}, \gamma_{2}\right)\right) \\
& =2-\sum_{n \in \mathbf{Z}} \mu\left(\pi_{n}\left(\gamma_{1}\right) \cap \pi_{n}\left(\gamma_{2}\right)\right)-1+\exp \left(-\sum_{n \in \mathbf{Z}} \lambda^{n} \nu\left(\pi_{n}\left(\gamma_{1}\right) \cap \pi_{n}\left(\gamma_{2}\right)\right)\right) \\
& =\frac{1}{2} \sum_{n \in \mathbf{Z}} \mu\left(\pi_{n}\left(\gamma_{1}\right) \triangle \pi_{n}\left(\gamma_{2}\right)\right)+\exp \left(-\sum_{n \in \mathbf{Z}} \lambda^{n} \nu\left(\pi_{n}\left(\gamma_{1}\right) \cap \pi_{n}\left(\gamma_{2}\right)\right)\right) .
\end{aligned}
$$

Thus $\mathscr{P}_{\lambda}$ is a Polish space with respect to the metric $r$ given by

$$
r(\varpi, \eta)=\sum_{n \in \mathbf{Z}} \mu\left(\varpi_{n} \Delta \eta_{n}\right)+\exp \left(-\sum_{n \in \mathbf{Z}} \lambda^{n} \nu\left(\varpi_{n} \cap \eta_{n}\right)\right), \quad \varpi, \eta \in \mathscr{P}_{\lambda},
$$

and $\pi$ is the topological quotient mapping.

It is easy to handle that $\mathscr{P}_{\lambda}$ is contractible. Now we construct a continuous cross-section for $\pi$. Keeping in mind the standard decomposition of $X=\cup_{s \geq 0}[0$, $s$ ) we have the $\nu$-continuous decomposition $A=\cup_{s \in[0, \nu(A)]} A(s)$ for any measurable subset $A \subset X$. For a partition $\varpi=\left\{\varpi_{n}\right\}_{n \in \mathbf{Z}} \in \mathscr{P}_{\lambda}$ and $n \in \mathbf{Z}$ we have the disjoint decomposition $\theta_{\lambda}^{n} \varpi n=\bigcup_{k=1}^{\infty} A_{n, k}$, where

$$
A_{n, k}= \begin{cases}\left(\theta_{\lambda}^{n} \varpi_{n}\right)(k)-\left(\theta_{\lambda}^{n} \varpi_{n}\right)(k-1), & \text { if } k \leq \nu\left(\theta_{\lambda}^{n} \varpi_{n}\right) \\ \theta_{\lambda}^{n} \varpi_{n}-\left(\theta_{\lambda}^{n} \varpi_{n}\right)(k-1), & \text { if } k-1<\nu\left(\theta_{\lambda}^{n} \varpi_{n}\right)<k \\ \varnothing, & \text { otherwise. }\end{cases}
$$

So, $\nu\left(\mathrm{A}_{n, k}\right) \leq 1$ for all $n, k$. Let $\beta$ be a bijection $\mathbf{N} \rightarrow \mathbf{Z} \times \mathbf{N}$. We put $a_{l}=\nu\left(A_{\beta(l)}\right)$. Since $\varpi \in \mathscr{P}_{\lambda}$, it follows that $\sum_{l} a_{l}=\infty$. We put $s_{0}=0, s_{n}=a_{1}+\cdots+a_{n}, B_{n}=\left[s_{n-1}\right.$, $\left.s_{n}\right)$ for $n \in \mathbf{N}$. Then $X=\cup_{n} B_{n}$ and $\nu\left(B_{n}\right)=\nu\left(A_{\beta(n)}\right)$. By Lemma 2.4 there is a partial isomorphism $\delta\left(A_{\beta(n)}, B_{n}\right) \in[\Delta]_{*}$ with $\operatorname{Dom}\left(\delta\left(A_{\beta(n)}, B_{n}\right)\right)=A_{\beta(n)}$, $\operatorname{Im}\left(\delta\left(A_{\beta(n)}, B_{n}\right)\right)=B_{n}$ and such that the continuity property (ii) of Lemma 2.4 is satisfied. Now we set

$$
\tau(\varpi) x=\delta\left(A_{\beta(k)}, B_{k}\right) \theta_{\lambda}^{n} x \quad \text { for } x \in \theta_{\lambda}^{-n} A_{n, k}, \quad(n, k) \in \mathbf{Z} \times \mathbf{N} .
$$

It is a routine to check that the automorphism $\tau(\varpi) \in[\Gamma]$, the map

$$
\tau: \mathscr{P}_{\lambda} \ni \varpi \mapsto \tau(\varpi) \in[\Gamma]
$$


is continuous, and $\pi \circ \tau=\mathrm{id}$, as desired. It follows that $[\Gamma]$ is homeomorphic to the product $[\Delta] \times \mathscr{P}_{\lambda}$. Since both multiples are contractible, the theorem is done.

Proof of the following theorem can be organized in a similar way.

Theorem 2.8. Let $[\Gamma]$ be an ergodic transformation group of type $I I I_{1}$. If there exists a strictly $\Gamma$-admissible, $\mu$-equivalent measure, then $[\Gamma]$ endowed with the uniform topology is contractible.

So, if $\Gamma$ is approximately finite, or $\Gamma=\Gamma_{1} \times \Gamma_{2}$, where $\Gamma_{i}$ is an ergodic type $I I I_{i}, 0<\lambda_{i}<1$, and $\log \lambda_{1}$ and $\log \lambda_{2}$ are rationally independent, then $[\Gamma]$ is contractible.

Note that Theorems 2.7 and 2.8 can be easily generalized to the nonergodic case, i.e. where $\Gamma$ can be represented in the form of a measurable field of ergodic transformation groups of type $I I I_{\lambda}, 0<\lambda \leq 1$.

2.4. Throughout this subsection $\Gamma$ is assumed to be ergodic and of type $I I I_{0}$. All other cases were considered above. We need to remind some definitions.

Two countable groups $\Gamma_{1} \subset$ Aut $\left(X_{1}, \mu_{1}\right)$ and $\Gamma_{2} \subset$ Aut $\left(X_{2}, \mu_{2}\right)$ are called weakly equivalent if there is a one-to-one measurable map $\varphi: X_{1} \rightarrow X_{2}$ such that measures $\mu_{2}$ and $\mu_{1} \circ \varphi^{-1}$ are equivalent and $\varphi\left[\Gamma_{1}\right] \varphi^{-1}=\left[\Gamma_{2}\right]$. Note, that then $\left[\Gamma_{1}\right]$ and $\left[\Gamma_{2}\right]$ endowed with the uniform topology are isomorphic as topological groups.

Consider the Haar measure $\chi_{\mathbf{z}}$ on the group $\mathbb{Z}: \chi_{\mathbf{z}}(i)=1, i \in \mathbb{Z}$. Denote by $\tau$ the shift on $\mathbb{Z}: \tau(i)=i+1$. For a countable group $\Delta \subset$ Aut $(X, \mu)$, the product $\tilde{\delta}$ $=\delta \times\left\{\left.\tau^{n}\right|_{n \in \mathbb{Z}}\right\}$ will be called the countable expansion of $\Delta$. It is well known that $\Delta$ is weakly equivalent to $\Delta$ if $\Delta$ is ergodic and infinite type (i.e. not type $I I_{1}$ ).

Let $G$ be a Polish group. For a countable group $\Sigma \subset$ Aut $(X, \mu)$ we denote by $\mathscr{R}(\Sigma) \subset X \times X$ the $\Sigma$-orbital equivalence relation. A measurable map $\alpha: \mathscr{R}(\Sigma) \rightarrow G$ is an orbital cocycle if $\alpha(x, y) \alpha(y, z)=\alpha(x, y)$ for a.e. $(x, y),(y, z) \in \mathscr{R}(\Sigma)$. An orbital cocycle $\alpha$ is said to be transient if there are a neighbourhood $V$ of the identity in $G$ and a subset $A \subset X$ with $\mu(A)>0$ and $\alpha(x, y) \notin V$ for all $(x, y) \in$ $\mathscr{R}(\Sigma) \cap(A \times A), x \neq y[\mathrm{Sh} 1]$.

First of all we prove the discrete decomposition theorem for $\Gamma$ which generalizes the Krieger's theorem for approximately finite groups $[\mathrm{Kr} 2, \mathrm{HO}]$. Since $\Gamma$ is of type $I I I_{0}$, there exist a $\mu$-equivalent measure $\nu$ and a neighbourhood $V$ of the identity in $\mathbb{R}_{+}^{*}$ with $\nu(X)=\infty$ and $\frac{d \nu \circ \gamma}{d \nu}(x) \notin V-\{1\}$ at a.e. $x \in X$ for all $\gamma$ $\in \Gamma[\mathrm{HO}, \mathrm{S} 1]$. By $[\mathrm{FM}]$ there is a (nonergodic) countable subgroup $\Delta \subset \mathrm{Aut}(X$, $\mu$ ) with 


$$
[\Delta]=\left\{\gamma \in[\Gamma] \mid \frac{d \nu \circ \gamma}{d \nu}(x)=1 \quad \text { for a.e. } \quad x \in X\right\} .
$$

Let $p: X \rightarrow Z$ be the $\Delta$-ergodic decomposition and $\kappa=\mu \circ p^{-1}$. According to [S1, Theorems 7.22, 8.7 and Corollary 6.9] the following properties are satisfied:

(i) there is a measurable field $Z \ni z \mapsto \nu_{z}$ of $\sigma$-finite, nonatomic, $\Delta$-invariant measures on $(X, \mathfrak{B})$ with $\nu_{z}\left(X-p^{-1}(z)\right)=0$ and $\nu=\int_{Z} \nu_{z} d \kappa(z)$;

(ii) there is an ergodic transformation $Q \in \operatorname{Aut}(Z, \kappa)$ with

$$
p(\Gamma x)=\left\{Q^{n} p(x) \mid n \in \mathbb{Z}\right\} \quad \text { for a.e. } \quad x \in X
$$

(iii) there is a transient orbital cocycle $\alpha: \mathscr{R}(Q) \rightarrow \mathbf{R}_{+}^{*}$ with

$$
\frac{d \nu \circ \gamma}{d \nu}(x)=\alpha(p(x), p(\gamma x)) \quad \text { at a.e. } \quad x \in X \quad \text { for all } \gamma \in \Gamma \text {. }
$$

We fix a Lebesgue space $(Y, \lambda)$ with a nonatomic $\sigma$-finite measure $\lambda$ and its partition $\left\{Y_{n}\right\}_{n=1}^{\infty}$ such that $\lambda\left(Y_{n}\right)=\infty, n \in \mathbf{N}$. It follows from (i) that there is a measurable field $Z \ni z \mapsto P_{z}$ of isomorphisms $P_{z}: Y \rightarrow p^{-1}(z)$ with $\nu_{z} \circ P_{z}=\lambda$. Then the map

$$
P: Z \times Y \ni(z, y) \mapsto P_{z}(y) \in p^{-1}(z) \subset X
$$

transforms $Z \times Y$ onto $X$ and the measure $\nu \circ P$ to $\kappa \times \lambda$. For simplicity we will identify $(X, \nu)$ with $(Z \times Y, \kappa \times \lambda)$ and $\Gamma$ with $P^{-1} \Gamma P$. It follows that $p(z, y)=z$ for a.e. $(z, y) \in X$. So, for every automorphism $\delta \in \Delta$ there is a measurable field $Z \ni z \rightarrow \delta_{z} \in \operatorname{Aut}_{0}(Y, \lambda)$ of $\lambda$-preserving transformations such that

$$
\delta(z, y)=\left(x, \delta_{z} y\right) \quad \text { for a.e. }(z, y) \in X
$$

and the groups $\Delta_{z}=\left\{\delta_{z} \mid \delta \in \Delta\right\}$ are ergodic and of type $I I_{\infty}$ for a.a. $z \in Z$.

Choose two sequences $\left\{B_{n}\right\}_{n=1}^{\infty}$ and $\left\{\gamma_{n}\right\}_{n=1}^{\infty}$ of measurable subsets of $X$ and elements of $\Gamma$ respectively in such a way that $\left\{p\left(B_{n}\right)\right\}_{n=1}^{\infty}$ is a partition of $Z$, $\lambda\left(\left\{y \mid(z, y) \in B_{n}\right\}\right)>0$ for all $z \in p\left(B_{n}\right)$, and $p\left(\gamma_{n} x\right)=Q p(x)$ for a.e. $x \in B_{n}$. By re placing, if necessarily, $\Gamma$ by its countable expansion, we may assume that

$$
\lambda\left(\left\{y \in Y \mid(z, y) \in B_{n}\right\}\right)=\infty=\lambda\left(\left\{y \in Y \mid(\hat{z}, y) \in \gamma_{n} B_{n}\right\}\right)
$$

for all $z \in \pi\left(B_{n}\right)$ and $\hat{z} \in \pi\left(\gamma_{n} B_{n}\right), n \in \mathbf{N}$. It follows from Lemma 2.6 that there are partial isomorphisms $\delta_{n} \hat{\delta}_{n} \in[\Delta] *$ with $\operatorname{Dom}\left(\delta_{n}\right)=\operatorname{Im}\left(\hat{\delta}_{n}\right)=Z \times Y_{n}, \operatorname{Im}\left(\hat{\delta}_{n}\right)$ 
$=B_{n}$, and $\operatorname{Dom}\left(\hat{\delta}_{n}\right)=\gamma_{n} B_{n}, n \in \mathbb{N}$. We define an automorphism $Q_{0} \in[\Gamma]$ by setting

$$
Q_{0}(z, y)=\hat{\delta}_{n} \gamma_{n} \delta_{n}(z, y) \quad \text { for all }(z, y) \in Z \times Y_{n}, n \in \mathbb{N}
$$

Since $\pi\left(Q_{0} x\right)=Q \pi(x)$ for a.e. $x \in X$, there exists a measurable field $Z \ni z \mapsto U_{z}$ $\in \operatorname{Aut}(Y, \lambda)$ of automorphisms of $(Y, \lambda)$ with

$$
Q_{0}(z, y)=\left(Q z, U_{z} y\right) \quad \text { for a.e. }(z, y) \in X
$$

Using (iii) we obtain that $\frac{d \nu \circ\left(Q_{0}^{-1} \delta Q_{0}\right)}{d \nu}(x)=1$ at a.e. $x$ for all $\delta \in \Delta$. Therefore $Q_{0}^{-1} \delta Q_{0} \in[\Delta]$, i.e. $Q_{0} \in N[\Delta]$. This together with (2-3) leads to $U_{z}\left[\Delta_{z}\right] U_{z}^{-1}$ $=\left[\Delta_{Q z}\right]$ for $\kappa$-a.e. $z \in Z$. Since $\Delta_{z}$ is ergodic, the function $Y \ni y \mapsto \frac{d \lambda \circ U_{z}}{d \lambda}(y)$ is almost everywhere a constant which will be denoted by mod $U_{z}$ (cf. $[\mathrm{CK}]$ ).

By using (ii) and (iii), one can prove that the full group $[\Gamma]$ is generated by $\Delta$ and $Q_{0}$. Thus, we have proved the following structure theorem (cf. [B]).

Theorem 2.9. Let $\Gamma$ be as above. Then the full group $[\Gamma]$ is generated by the actions (2-2) and (2-3) of $\Delta$ and $Q_{0}$ respectively on $(Z \times Y, \kappa \times \lambda), Q_{0} \in N[\Delta]$. Every automorphism $\delta \in \Delta$ preserves $\kappa \times \lambda$ and

$$
\frac{d(\kappa \times \lambda) \circ Q_{0}}{d(\kappa \times \lambda)}(z, y)=\varphi(z)
$$

for some function $\varphi: Z \rightarrow \mathbb{R}_{+}^{*}-V$, where $V$ is a neighbourhood of the identity in $\mathbb{R}_{+}^{*}$.

Note that $\varphi(z)=\frac{d \kappa \circ Q}{d \kappa}(z) \cdot \bmod U_{z}$, and the orbital cocycle

$$
\Phi: \mathscr{R}(Q) \ni(z, w) \mapsto \Phi(z, w) \in \mathbb{R}_{+}^{*}
$$

determined by the correlation $\Phi(z, Q z)=\phi(z), z \in Z$, is transient.

Theorem 2.10. Let $\Gamma$ be as above. Then the full group $[\Gamma]$ endowed with the uniform topology is contractible.

Proof. We use the notations of the previous theorem. Moreover, we assume that $Y=[0, \infty), \lambda$ is a Lebesgue measure on $Y$, and $\lambda_{0}$ is the $\lambda$-equivalent probability measure with $d \lambda_{0}(y)=\exp (-y) d \lambda(y)$ for all $y \in Y$. Let 


$$
U: R(Q) \ni(z, w) \mapsto U(z, w) \in \operatorname{Aut}(Y, \lambda)
$$

be the orbital cocycle such that $U(z, Q z)=U_{z}$ for all $z \in Z$. Denote by $\mathscr{P}_{0}$ the family of countable partitions $\varpi=\left\{\varpi_{n}\right\}_{n \in \mathbf{Z}}$ of $X$ with

$$
\sum_{n \in \mathrm{Z}} \lambda\left(\varpi_{n}\left[Q^{-n} z\right]\right) \cdot \bmod U\left(Q^{-n} z, z\right)=\infty \quad \text { for } \kappa^{- \text {a.e. }} \quad z \in Z
$$

Here the measurable field $Z \ni z \mapsto \varpi[z]=\left\{\varpi_{n}[z]\right\}_{n \in \mathbf{Z}}$ of $Y$-partitions is deter-

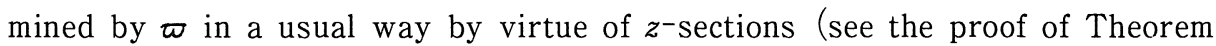
$2.2)$. Note that $(2-5)$ is equivalent to the fact that $\sum_{n \in \mathbf{Z}} \lambda\left(\left\{y \mid(z, y) \in Q_{0}^{n} \varpi_{n}\right\}\right)=$ $\infty$ at a.e. $z$.

For a transformation $\theta \in[\Gamma]$ we define a partition $\pi(\theta)=\left\{\pi_{n}(\theta)\right\}_{n \in \mathbf{Z}}$ by setting

$$
x \in \pi_{n}(\theta) \Longleftrightarrow \theta x=\delta_{n} Q_{0}^{n} x \text { for some automorphism } \delta_{n} \in[\Delta]
$$

Since $\left\{\theta \pi_{n}(\theta)\right\}_{n \in \mathbf{Z}}$ is a partition of $X$ and $\Delta$ preserves $\kappa \times \lambda$, we have $\pi(\theta) \in$ $\mathscr{P}_{0}$. Moreover, the map

$$
[\Gamma] \ni \theta \mapsto \pi(\theta) \in \mathscr{P}_{0}
$$

is continuous and $\pi(\delta \theta)=\pi(\theta)$ for every transformation $\delta \in[\Delta]$. Note, that $[\Delta]$ is a closed subgroup of $\mathscr{P}_{0}$. We endow $\mathscr{P}_{0}$ with metric $r$ given by

$$
\begin{aligned}
r(\varpi, \eta) & =\sum_{n \in \mathbf{Z}}\left(\kappa \times \lambda_{0}\right)\left(\varpi_{n} \Delta \eta_{n}\right) \\
& +\int_{Z}^{\exp }\left(-\sum_{n \in \mathbf{Z}} \lambda\left(\varpi_{n}[z] \cap \eta_{n}[z]\right) \cdot \bmod U\left(Q^{-n} z, z\right)\right) d \kappa(z)
\end{aligned}
$$

for all $\varpi, \eta \in \mathscr{P}_{0}$. Like it was done in Theorem 2.7 for a type $I I I_{\lambda}$ transformation group, one can prove that $\left(\mathscr{P}_{0}, r\right)$ is homeomorphic to the quotient space $[\Delta] \backslash$ $[\Gamma]$.

Now prove the contractibility of $\mathscr{P}_{0}$. Let $\mathscr{P}(Y)$ be the family of countable partitions of $\left(Y, \lambda_{0}\right)$ and $\widetilde{r}$ the natural Polish metric on it:

$$
\widetilde{r}(\varpi, \eta)=\sum_{n \in \mathrm{Z}} \lambda_{0}\left(\varpi_{n} \Delta \eta_{n}\right), \quad \varpi, \eta \in \mathscr{P}(Y) .
$$

There exists a continuous map

$$
\sigma: \mathscr{P}(Y) \times[0,+\infty) \ni(\varpi, t) \mapsto \sigma_{t}(\varpi)=\left\{\sigma_{t}(\varpi)_{n}\right\}_{n \in \mathbf{Z}} \in \mathscr{P}(Y)
$$

with 
(i) $\sigma_{0}(\varpi)=\varpi, \sigma_{\infty}(\varpi)_{n}=\varnothing$ for all $n \neq 0$, and $\sigma_{\infty}(\varpi)_{0}=X$;

(ii) for each $l \in \mathbb{Z}_{+}$there exists $\mathrm{m}(l) \in \mathbb{Z}-\{0\}$ such that if a nonzero integer $n$ is not equal to $m(l)$ then $\sigma_{t}(\varpi)_{n}=\sigma_{l}(\varpi)_{n}$ for all $t \in[l, l+1)$.

(iii) $\lambda\left(\varpi_{m(l)} \Delta \sigma_{t}(\varpi)_{m(l)}\right)<\infty$ for all $t \in[l, l+1)$ and $n \in \mathbb{Z}$.

Now for a partition $\varpi=(Z \ni z \rightarrow \varpi[z]) \in \mathscr{P}_{0}$ we let $\tilde{\sigma}_{t}(\varpi)=(Z \ni z \mapsto$ $\left.\sigma_{t}(\varpi[z])\right)$. It follows from (ii) and (iii) that $\tilde{\sigma}_{t}(\varpi) \in \mathscr{P}_{0}$ for all $t>0$ and the map

$$
\widetilde{\sigma}: \mathscr{P}_{0} \times[0,+\infty) \mapsto \widetilde{\sigma}_{t}(\varpi) \in \mathscr{P}_{0}
$$

is continuous. So we deduce from (i) that $\widetilde{\sigma}$ is a contraction path on $\mathscr{P}_{0}$.

To construct a continuous map $\tau: \mathscr{P}_{0} \rightarrow[\Gamma]$ with $\pi \circ \tau=\mathrm{id}$ one should repeat almost literally the final part of the proof of Theorem 2.7 replacing $\theta_{\lambda}$ by $Q_{0}$ and using Lemma 2.5 instead of Lemma 2.4.

Thus, $[\Gamma]$ is homeomorphic to the Cartesian product $\mathscr{P}_{0} \times[\Delta]$. Since $[\Delta]$ is contractible by Theorem 2.2 , the contractibility of $[\Gamma]$ follows.

\section{§3. Homotopical Properties of the Normalizers of Full Groups}

3.1. Throughout this section $\Gamma=\left\{\gamma_{k}\right\}_{k=1}^{\infty}$ is an ergodic approximately finite group of automorphisms of $(X, \mathfrak{B}, \mu), \mu(X)=1$. Let $\tilde{\mu}$ be a measure on the $\Gamma$-orbital equivalence relation $(\mathscr{R},(\mathfrak{B} \times \mathfrak{B}) \backslash \mathscr{R}(\Gamma))$ defined by $d \tilde{\mu}(x, y)=d \mu(x)$. We consider two maps:

$$
\begin{aligned}
& \pi: \mathscr{R}(\Gamma) \ni(x, y) \mapsto x \in X \\
& \sigma: \mathscr{R}(\Gamma) \ni(x, y) \mapsto(y, x) \in \mathscr{R}(\Gamma) .
\end{aligned}
$$

Let $\hat{\mu}$ be a $\tilde{\mu}$-equivalent probability measure with $\pi(\hat{\mu})=\mu$. Note that $\sigma$ belongs to $\operatorname{Aut}(\mathscr{R}(\Gamma), \hat{\mu})$. We set $\mathscr{H}=L^{2}(\mathscr{R}(\Gamma), \hat{\mu})$ and define $\mathscr{P}_{+}(\mathscr{H})=\left\{P_{A} \mid A \in(\mathfrak{B} \times \mathfrak{B})\right.$ $\lceil\mathscr{R}(\Gamma)\}, \mathcal{U}(\mathscr{H}), \mathcal{U}_{+}(\mathscr{H})$ just in the same manner as in Section 1 . So we have an anti-isomorphism of topological groups:

$$
\operatorname{Aut}(X, \mu) \ni \theta \mapsto U_{\theta} \in U_{+}(\mathscr{H}) \text {. }
$$

It is easy to verify that the Boolean subalgebra of projectors

$$
\mathscr{P}_{++}=\left\{P_{\pi^{-1} A} \mid A \in \mathfrak{B}\right\}
$$

is a closed subset of $\mathscr{P}_{+}(\mathscr{H})$. Therefore 


$$
U_{++}=\left\{U \in \mathscr{U}_{+}(\mathscr{H}) \mid U \mathscr{P}_{++} U^{*}=\mathscr{P}_{++} \text {and } U U_{\sigma}=U_{\sigma} U\right\}
$$

is a closed subgroup of $U_{+}(\mathscr{H})$.

For a transformation $\theta \in N[\Gamma]$ we let

$$
i(\theta)(x, y)=(\theta x, \theta y), \quad(x, y) \in \mathscr{R}(\Gamma)
$$

Then $i(\theta) \in$ Aut $(\mathscr{R}(\Gamma), \tilde{\mu})$, the map $N[\Gamma] \ni \theta \mapsto i(\theta)$ is a one-to-one homomorphism, and $U_{i(\theta)} \in U_{++}$. We shall prove that $U_{i(N[\Gamma])}=U_{++}$. Actually, suppose that a unitary $U$ belongs to $\mathcal{U}_{++}$. Since $U \in \mathcal{U}_{+}(\mathscr{H})$, there is a measurable transformation $\theta \in \operatorname{Aut}(\mathscr{R}(\Gamma), \hat{\mu})$ with $U_{\theta}=U$ (see Section 1 ). We write $\theta$ as

$$
\theta(x, y)=(A(x, y), B(x, y)), \quad(x, y) \in \mathscr{R}(\Gamma)
$$

where $A$ and $B$ are two measurable functions from $\mathscr{R}(\Gamma)$ onto $X$. Since $U_{\theta} \mathscr{P}_{++} U_{\theta}^{*}$ $=\mathscr{P}_{++}$, there exists an automorphism $\vartheta \in \operatorname{Aut}(X, \mu)$ with $A(x, y)=\vartheta x$ for $\hat{\mu}-$ a.e. $(x, y) \in \mathscr{R}(\Gamma)$. Finally, the condition $U_{\theta} U_{\sigma}=U_{\sigma} U_{\theta}$ implies $B(x, y)=\vartheta y$ for $\hat{\mu}$-a.e. $(x, y)$. Thus we may assume that

$$
\theta(x, y)=(\vartheta x, \vartheta y), \quad \text { for } \hat{\mu} \text {-a.e. }(x, y) \in \mathscr{R}(\Gamma)
$$

Since $\theta$ takes $\hat{\mu}$ to an equivalent measure, we have $\vartheta \in N[\Gamma]$, as desired.

So the map $N[\Gamma] \ni \theta \mapsto U_{i(\theta)} \in U_{++}$is an algebraic anti-isomorphism. We furnish $N[\Gamma]$ with the topology in which this map is a homeomorphism. This topology will be called normal. Since $\boldsymbol{U}_{++}$is a closed subgroup of $\mathcal{U}(\mathscr{H})$, it follows that $N[\Gamma]$ is a Polish group.

We shall show that the normal topology is compatible with the metric $d$ introduced by T. Hamachi and M. Osikawa [HO]:

$$
d(\theta, \zeta)=d_{w}(\theta, \zeta)+\sum_{k=1}^{\infty} \frac{1}{2^{k}} \frac{d_{u}\left(\theta^{-1} \gamma_{k} \theta, \zeta^{-1} \gamma_{k} \zeta\right)}{1+d_{u}\left(\theta^{-1} \gamma_{k} \theta, \zeta^{-1} \gamma_{k} \zeta\right)}, \quad \theta, \zeta \in N[\Gamma]
$$

For every automorphism $\gamma \in[\Gamma]$ consider its graph

$$
\mathfrak{g}(\gamma)=\{(x, \gamma x) \mid x \in X\} \subset \mathscr{R}(\Gamma)
$$

Then $\mathfrak{g}(\gamma)$ is measurable and $\hat{\mu}(\mathfrak{g}(\gamma))>0$. Suppose that a sequence $\theta_{n} \in N[\Gamma]$, $n \in \mathbf{N}$, normally converges to an automorphism $\theta \in N[\Gamma]$. Then $\left\{i\left(\theta_{n}\right)\right\}_{n=1}^{\infty} \in$ Aut $(\mathscr{R}(\Gamma), \hat{\mu})$ weakly converges to the transformation $i(\theta) \in$ Aut $(\mathscr{R}(\Gamma), \hat{\mu})$. Hence we have 


$$
\begin{gathered}
\hat{\mu}\left(i\left(\theta_{n}\right) \pi^{-1}(A) \triangle_{i}\left(\theta_{n}\right) \pi^{-1}(A)\right) \rightarrow 0 \text { as } n \rightarrow \infty \text { for every } A \in \mathfrak{B}, \\
\hat{\mu}\left(i\left(\theta_{n}\right) \mathfrak{g}(\gamma) \triangle_{i}\left(\theta_{n}\right) \mathfrak{g}(\gamma)\right) \rightarrow 0 \text { as } n \rightarrow \infty \text { for every } \gamma \in \mathbb{\Gamma} .
\end{gathered}
$$

The first is equvalent to $\mu\left(\theta_{n} A \triangle \theta A\right) \rightarrow 0$ for every $A \in \mathfrak{B}$, i.e. $d_{w}\left(\theta_{n}, \theta\right) \rightarrow 0$ as $n$ $\rightarrow \infty$. The second implies that $d_{u}\left(\theta_{n} \gamma \theta_{n}^{-1}, \theta \gamma \theta^{-1}\right) \rightarrow 0$ as $n \rightarrow \infty$. Thus $d\left(\theta_{n}, \theta\right) \rightarrow$ 0 . It follows that the normal topology is stronger than the Hamachi-Osikawa one. Since $(N[\Gamma], d)$ is also a Polish group [HO], the open mapping theorem yields that these topologies are equivalent.

The main goal of this section is to study the homotopical structure of $N[\Gamma]$ endowed with the normal topology.

3.2. The following statement is the measure theoretical analogue of [PT, Theorem 4].

Theorem 3.1. If $\Gamma$ is of type $I I_{1}$ then $N[\Gamma]$ is contractible.

Proof. Since all approximately finite ergodic transformation groups are pairwise weakly equivalent, we may assume that

(i) $X=\{0,1\}^{\mathbf{N}}$ and the measure $\mu=\otimes_{n=1}^{\infty} \nu$, where $\nu$ is the following measure on $\{0,1\}: \nu(0)=\nu(1)=1 / 2$;

(ii) the group $\Gamma$ is generated by automorphisms $\delta_{i}, i \in \mathbf{N}$ :

$$
\left(\delta_{i} x\right)_{j}= \begin{cases}x_{i}+1(\bmod 2), & \text { if } j=i \\ x_{j}, & \text { otherwise }\end{cases}
$$

For every multi-index $\alpha=\left(\alpha_{1}, \ldots, \alpha_{n}\right)$ of the length $n$ we set $X(\alpha)=\left\{x=\left(x_{i}\right)_{i} \in\right.$ $\left.X \mid x_{i}=\alpha_{i}, i=1, \ldots, n\right\}$ and $\gamma(\alpha)=\delta_{1}^{\alpha_{1}} \delta_{2}^{\alpha_{2}} \ldots \delta_{n}^{\alpha_{n}}$. Then $\gamma(\alpha) X(\varepsilon(n))=X(\alpha)$, where $\varepsilon(n)=(0, \ldots, 0)$.

Take an automorphism $\theta \in N[\Gamma]$. Since $\theta$ preserves $\mu$, for every $n \in N$ there exists a partial isomorphism $s_{n}(\theta) \in[\Gamma] *$ with $\operatorname{Dom}\left(s_{n}(\theta)\right)=X(\varepsilon(n))$ and $\operatorname{Im}\left(s_{n}(\theta)\right)=\theta X(\varepsilon(n))$. Moreover, by Lemma 2.4 one can make the map $N[\Gamma] \ni \theta$ $\mapsto s_{n}(\theta) \in[\Gamma]_{*}$ continuous. Remind that the groupoid $[\Gamma] *$ is endowed with the uniform topology. Now we define a transformation $\widetilde{s}_{n}(\theta) \in[\Gamma]$ by setting

$$
\widetilde{s_{n}}(\theta) x=\theta \gamma(\alpha) \theta^{-1} s_{n}(\theta) \gamma(\alpha)^{-1} x \quad \text { for all } x \in X(\alpha), \alpha \in\{0,1\}^{n}
$$

Then the map $N[\Gamma] \ni \theta \mapsto \widetilde{s_{n}}(\theta) \in[\Gamma]$ is continuous, $\widetilde{s_{n}}(\theta) X(\alpha)=\theta X(\alpha)$, and the commutator

$$
\left[\theta^{-1} \widetilde{s}_{n}(\theta), \gamma(\alpha)\right]=\theta^{-1} \widetilde{s}_{n}(\theta) \gamma(\alpha) \widetilde{s}_{n} \theta^{-1} \theta \gamma(\alpha)^{-1}=\mathrm{id}
$$


Therefore $d\left(\widetilde{s}_{n}(\theta), \theta\right) \rightarrow 0$ as $n \rightarrow \infty$.

Let $\left\{\beta_{t}^{(n)} \mid t \in[0,1]\right\}$ be a contraction path for the induced full group $[\Gamma]_{X(\varepsilon(n))}$ with $\beta_{0}^{(n)}(\gamma)=\gamma$ and $\beta_{1}^{(n)}(\gamma)=\mathrm{id}\left\lceil X(\varepsilon(n)), n \in \mathbf{Z}_{+}\right.$. Notice, that we put $X(\varepsilon(0))=X$.

For each $n \in \mathbf{Z}_{+}$and $t \in[0,1]$ we define an automorphism $\partial_{t}^{(n)}(\theta) \in[\Gamma]$ by setting

$\partial_{t}^{(n)}(\theta) x=\gamma(\alpha) \beta_{t}^{(n)}\left(\widetilde{s}_{n+1}(\theta)^{-1} s_{n}(\theta)\right) \gamma(\alpha)^{-1} x$, for all $x \in X(\alpha), \alpha \in\{0,1\}^{n}$, where $s_{0}(\theta)=\mathrm{id}$. Then the map

$$
\partial^{(n)}: N[\Gamma] \times[0,1] \ni(\theta, t) \mapsto \partial_{t}^{(n)}(\theta) \in[\Gamma]
$$

is continuous, $\partial_{0}^{(n)}(\theta)=\widetilde{s}_{n+1}(\theta)^{-1} s_{n}(\theta), \partial_{1}^{(n)}(\theta)=\mathrm{id}$. Moreover, $\partial_{t}^{(n)}(\theta) X(\alpha)=$ $X(\alpha),\left[\partial_{t}^{(n)}(\theta), \gamma(\alpha)\right]=$ id for every $\alpha \in\{0,1\}^{n}$ and $t \in[0,1]$. Therefore $d\left(\partial_{t}^{(n)}(\theta)\right.$, id $) \rightarrow 0$ as $n \rightarrow \infty$.

Now for each $n \in \mathbb{Z}_{+}$and $t \in[0,1)$ we set

$$
\sigma_{n+1}(\theta)=\left(\widetilde{s}_{n+1}(\theta) \partial_{t}^{(n)}(\theta)\right)^{-1} \theta
$$

It is straightforward to check that the map

$$
\sigma: N[\Gamma] \times[0,+\infty) \ni(\theta, u) \mapsto \sigma_{u}(\theta) \in N[\Gamma]
$$

is continuous, $\sigma_{0}(\theta)=\theta$, and $\sigma_{\infty}(\theta)=\mathrm{id}$. This means that $N[\Gamma]$ is contractible.

By $\mathrm{Cl}_{d}([\Gamma])$ we denote the closure of $[\Gamma]$ in the normal topology, i.e. the group of approximately inner automorphisms.

Theorem 3.2. If $\Gamma$ is of type $I_{\infty}$, then $N[\Gamma]$ and $\mathrm{Cl}_{d}([\Gamma])$ are contractible.

Proof. We have a split exact sequence of Polish groups

$$
1 \longrightarrow \mathrm{Cl}_{d}([\Gamma]) \longrightarrow N[\Gamma] \stackrel{\bmod }{\longrightarrow} \mathbb{R}_{+}^{*} \longrightarrow 1
$$

Thus $N[\Gamma]$ is precisely the semidirect product of $\mathrm{Cl}_{d}([\Gamma])$ and $\mathbf{R}_{+}^{*}$, so that its topological structure is completely determined by that of $\mathrm{Cl}_{d}([\Gamma])$.

Let $\nu$ be a $\mu$-equivalent, $\Gamma$-invariant measure, $\nu(X)=\infty$. Then an automorphism $\theta \in N[\Gamma]$ is approximately inner if and only if $\nu \circ \theta=\nu$. So, only a slight modification of the proof of Theorem 3.1 is needed to prove the contractibility of $\mathrm{Cl}_{d}([\Gamma])$. 
Note that the requirement for $\Gamma$ to be approximately finite in Theorem 3.1 can not be dropped. The paper [GeG] contains a family of examples of ergodic type $I I$ transformation groups $\Gamma$ with the noncontractible (or even nonconnected) normalizers $N[\Gamma]$. However, for all of them $\mathrm{Cl}_{d}([\Gamma])=[\Gamma]$. Therefore by the open mapping theorem for Polish groups the normal topology $d$ on $N[\Gamma]$ induces the uniform topology on $\mathrm{Cl}_{d}([\Gamma])$. It follows that $\mathrm{Cl}_{d}([\Gamma])$ is contractible just as in the approximately finite case. We do not know if this is still true for any ergodic type $I I_{1}$ transformation group.

3.3. Let $\Gamma$ be of type $I I I_{\lambda}, 0<\lambda<1$. Then we have a split exact sequence

$$
1 \longrightarrow \mathrm{Cl}_{d}([\Gamma]) \longrightarrow N[\Gamma] \stackrel{\mathrm{mod}}{\longrightarrow} \mathbf{R}_{+}^{*} / \lambda^{\mathbf{Z}} \longrightarrow 1
$$

Like in the type $I_{\infty}$ case the topological structure of $\mathrm{Cl}_{d}([\Gamma])$ here determines that of $N[\Gamma]$. We shall prove the following

Theorem 3.3. $\quad \mathrm{Cl}_{d}([\Gamma])$ is contractible.

Proof. Let $X, \Gamma, X(\alpha), \gamma(\alpha)$ be as above (see the proof of Theorem 3.1); we only change the measure $\mu: \mu=\otimes_{n+1}^{\infty} \nu_{\lambda}$, where $\nu_{\lambda}$ is the following measure on $\{0,1\}: \nu_{\lambda}(0)=1 /(1+\lambda), \nu_{\lambda}(1)=\lambda /(1+\lambda)$. Donote by $\mathscr{P}_{\lambda}$ the family of countable partitions $\tau=\left\{\tau_{n}\right\}_{n \in \mathbf{Z}}$ of $X$ with $\sum_{n} \lambda^{n} \mu\left(\tau_{n}\right)=1$ and by $\Delta$ a countable transformation group with

$$
[\Delta]=\{\gamma \in[\Gamma] \mid \mu \circ \gamma=\mu\} \text {. }
$$

Then $\Delta$ is approximately finite, ergodic, and type $I_{1}$ and

$$
\mathrm{Cl}_{d}([\Delta])=\{\theta \in N[\Gamma] \mid \mu \circ \theta=\mu\}
$$

Since $\mathrm{Cl}_{d}([\Gamma])=\operatorname{Ker}(\bmod )[\mathrm{HO}]$, for each $\theta \in \mathrm{Cl}_{d}([\Gamma])$ we have $\frac{d \mu \circ \theta}{d \mu}(x) \in \lambda^{\mathbf{Z}}$ for $\mu$-a.e. $x$. So, the partition $\pi(\theta)=\left\{\pi_{n}(\theta)\right\}_{n \in \mathbf{Z}}$ defined by

$$
x \in \pi_{n}(\theta) \Longleftrightarrow \frac{d \mu \circ \theta}{d \mu}(x)=\lambda^{n}
$$

belongs to $\mathscr{P}_{\lambda}$. Just in the same way as in Theorem 2.7 , one can prove that $\mathscr{P}_{\lambda}$ endowed with the quotient topology is contractible and homeomorphic to the homogeneous space $\mathrm{Cl}_{n}([\Delta]) \backslash \mathrm{Cl}_{d}([\Gamma])$, and the quotient map $\pi: \mathrm{Cl}_{d}([\Gamma]) \ni \theta \mapsto$ $\pi(\theta) \in \mathscr{P}_{\lambda}$ admits a continuous cross-section. Thus it suffices to prove that the 
group $\mathrm{Cl}_{d}([\Delta])$ is contractible.

Since $\mu(\theta X(\varepsilon(n)))=\mu(X(\varepsilon(n)))$ for an automorphism $\theta \in \mathrm{Cl}_{d}([\Delta])$, there is a continuous map

$$
\mathrm{Cl}_{d}([\Delta]) \ni \theta \mapsto s_{n}(\theta) \in[\Delta] *
$$

with $\operatorname{Dom}\left(s_{n}(\theta)\right)=X(\varepsilon(n)), \operatorname{Im}\left(s_{n}(\theta)\right)=\theta X(\varepsilon(n)), n \in \mathbf{N}$. For each multi-index $\alpha \in\{0,1\}^{n}$ the function $X \ni x \mapsto \frac{d \mu \circ \gamma(\alpha)}{d \mu}(x)$ is constant on every cylinder set $X(\beta), \beta \in\{0,1\}^{n}$. Therefore, the transformation $\widetilde{s}_{n}(\theta) \in[\Gamma]$ determined by $(3-1)$ belongs to $[\Delta]$. We see that the arguments for the contractibility of $N[\Gamma]$ in the proof of Theorem 3.1 leads to the contractibility of $\mathrm{Cl}_{d}([\Delta])$.

Corollary 3.4. (i) $N[\Gamma]$ is homeomorphic to the Cartesian product $\mathrm{Cl}_{d}([\Delta])$ $\times \mathscr{P}_{\lambda} \times\left(\mathbf{R}_{+}^{*} / \lambda^{\mathbf{Z}}\right)$,

(ii) $\pi_{1}(N[\Gamma])=\mathbf{Z}$.

3.4. Now let $\Gamma$ be of type $I I I_{0}$. Denote by $W(\mathbf{R})=\{W(t)\}_{t \in \mathbf{R}}$ the associated (Poincaré) flow $[\mathrm{Kr} 2, \mathrm{HO}]$ acting on a space $(\Omega, \varrho)$ and by $C(W(\mathbf{R}))$ its centralizer, i.e.

$$
C(W(\mathbf{R}))=\{\zeta \in \operatorname{Aut}(\Omega, \varrho) \mid \zeta W(t)=W(t) \zeta \quad \text { for all } t \in \mathbf{R}\}
$$

It is known that $C(W(\mathbf{R}))$ endowed with the weak topology is a Polish group and the following sequence

$$
1 \longrightarrow \mathrm{Cl}_{d}([\Gamma]) \longrightarrow N[\Gamma] \stackrel{\bmod }{\longrightarrow} C(W(\mathbf{R})) \longrightarrow 1
$$

is exact and split $[\mathrm{H}]$.

Theorem 3.5. $\mathrm{Cl}_{d}([\Gamma])$ is contractible.

Proof. We use the notations of Theorems 2.9 and 2.10 and identify $\mu$ with $\kappa \times \lambda$. Since $\Delta$ is approximately finite, we may assume that $\Delta$ is generated by a $\mu$-preserving transformation $S_{0}$ :

$$
S_{0}(z, y)=(z, S y),(z, y) \in X
$$

where $S \in \operatorname{Aut}_{0}(Y, \lambda)$ is an ergodic transformation. It follows that $U_{z} \in N[S]$ for $\kappa$-a.e. $z$ (see $(2-3))$.

Take an automorphism $\theta \in \mathrm{Cl}_{d}([\Gamma])$. By [BG2, Proposition 1.5] there is a 
partition $\pi(\theta)=\pi_{n}(\theta)_{n \in \mathbf{Z}}$ of $X$ with

$$
\theta(z, y)=\left(Q^{n} z, \cdot\right) \text { and } \frac{d \mu \circ \theta}{d \mu}(x, y)=\frac{d \mu \circ Q_{0}^{n}}{d \mu}(x, y) \text { for a.e. }(z, y) \in \pi_{n}(\theta)
$$

It is staightforward that $\pi(\theta) \in \mathscr{P}_{0}$ (see the proof of Theprem 2.10) and map

$$
\pi: \mathrm{Cl}_{d}([\Delta]) \ni \theta \mapsto \pi(\theta) \in \mathscr{P}_{0}
$$

is continuous, since the set $\left\{\frac{d \mu \circ Q_{0}^{n}}{d \mu}(z, y) \mid n \in \mathbb{Z}\right\}$ is closed in $\mathbb{R}_{+}^{*}$ for $\mu$-a.e. $(z$, $y)$ in view of $(2-4)$. It can be proved that $\mathscr{P}_{0}$ is homeomorphic to the homogeneous space $\mathrm{Cl}_{d}([\Delta]) \backslash \mathrm{Cl}_{d}([\Gamma])$ and the quotient map $\pi$ admits a continuous cross-section. To this end one should apply the argument of the proof of Theorem 2.10. Since $\mathscr{P}_{0}$ is contractible, it suffices to prove that the group $\mathrm{Cl}_{d}([\Delta])$ is contractible.

Let $\Upsilon_{n}=\{0,1\}^{n} \times \mathbb{Z}$ and $\varepsilon(n)=(0, \cdots 0) \in \Upsilon_{n}, n \in \mathbb{N}$. For every $\alpha=\left(\alpha_{1}, \cdots\right.$, $\left.\alpha_{n+1}\right) \in \Upsilon_{n}$ we set $\alpha^{k}=\left(\alpha_{1}, \cdots, \alpha_{n}, k, \alpha_{n+1}\right) \in \Upsilon_{n+1}, k=1,2$. Since $\Gamma$ is approximately finite, there is a sequence of arrays $\left\{X(\alpha), \gamma(\alpha) \mid \alpha \in \Upsilon_{n}\right\}, n \in \mathbb{N}$, such that (see Theorem 2.9 and $[\mathrm{HO}]$ ):

(i ) $\{X(\alpha)\}_{\alpha \in Y_{n}}$ is a partition of $X, \mu(X(\alpha))>0$ for every $\alpha \in Y_{n}, n \in \mathbb{N}$

(ii) $\gamma(\alpha) \in[\Gamma]_{*}$ with $\operatorname{Dom}(\gamma(\alpha))=X(\varepsilon(n)), \operatorname{Im}(\gamma(\alpha))=X(\alpha)$,

(iii) $X(\alpha)=X\left(\alpha^{0}\right) \cup X\left(\alpha^{1}\right), \gamma\left(\alpha^{0}\right)\left(X\left(\alpha^{0}\right)\right)=X\left(\alpha^{1}\right)$ for all $\alpha \in \Upsilon_{n}$,

(iv) the collection of the sets $\left\{X(\alpha) \mid \alpha \in \Upsilon_{n}, n \in \mathbb{N}\right\}$ generates the $\sigma$-algebra $\mathfrak{B}$,

(v) $\cup_{n=1}^{\infty}\left[\Gamma_{n}\right]$ is uniformly dense in $[\Gamma]$, where $\Gamma_{n}$ is the automorphism group generated by the partial isomorphisms $\gamma(\alpha), \alpha \in \Upsilon_{n}$,

(vi) the function $X(\varepsilon(n)) \ni(z, y) \mapsto \frac{d \mu \circ \gamma(\alpha)}{d \mu}(z, y)$ is independent of $y$ for all $n \in \mathbb{N}$.

It is straightforward that for each automorphism $\theta \in \mathrm{Cl}_{d}([\Delta])$ there is a measurable field $Z \ni z \mapsto \theta(z) \in N[\Delta]$ of $\lambda$-preserving transformations of $Y$ with

$$
\theta(z, y)=(z, \theta(z) y),(z, y) \in X
$$

By applying Lemma 2.5 we conclude that for every $n \in \mathbb{N}$ there exists a partial isomorphism $s_{n}(\theta) \in[\Delta] *$ with $\operatorname{Dom}\left(s_{n}(\theta)\right)=X(\varepsilon(n)), \operatorname{Im}\left(s_{n}(\theta)\right)=\theta X(\varepsilon(n))$, and the map

$$
\mathrm{Cl}_{d}[\Delta] \ni \theta \mapsto s_{n}(\theta) \in[\Delta]_{*}
$$

is continuous. Now we define a transformation $\widetilde{s}_{n}(\theta) \in[\Gamma]$ by setting 


$$
\widetilde{s_{n}}(\theta)=\theta \gamma(\alpha) \theta^{-1} s_{n}(\theta) \gamma(\alpha)^{-1} x, \quad \text { for all } x \in X(\alpha), \alpha \in \gamma_{n}, n \in \mathbb{N}
$$

It follows from (vi) that $\mu \circ \widetilde{\mathrm{s}_{n}}(\theta)=\mu$, i.e. $\widetilde{s_{n}}(\theta) \in[\Delta]$. Moreover, by the definition of $\widetilde{s}_{n}(\theta)$ and $(\mathrm{i})-(\mathrm{iii})$ we have $\widetilde{s}_{n}(\theta) X(\alpha)=\theta X(\alpha)$ and $\left[\theta^{-1} \widetilde{s}_{n}(\theta), \gamma\right]=\mathrm{id}$ for all $\alpha \in \Upsilon_{n}, \gamma \in \Gamma_{n}$. Hence in view of (iv) and (v) $\widetilde{s}_{n}(\theta) \rightarrow \theta$ in the normal topology as $n \rightarrow \infty$. Let $\left\{\beta_{t}^{(n)}\right\}_{t \in[0,1]}$ be a contraction path for the induced full group $[\Delta]_{X(\varepsilon(n))}$ with $\beta_{0}^{(n)}(\delta)=\delta$ and $\beta_{1}^{(n)}(\delta)=$ id $\uparrow X(\varepsilon(n))$ for all $\delta \in$ $[\Delta]_{X(\in(n))}, n \in \mathbb{N}$ (see Theorem 2.2). The completion of the proof repeats almost literally that of Theorem 3.1 .

Corollary 3.6. Let $\Gamma$ be as above. The group $N[\Gamma]$ is homeomorphic to the Cartesian product $\mathrm{Cl}_{d}([\Delta]) \times \mathscr{P}_{0} \times C(W(\mathbb{R}))$.

\section{References}

[B] Bezuglyi, S. I., Groups of type $I I_{0}$ and some approximability conditions for groups of measure space transformations, preprint. 1981. (Russian).

[BG1] Bezuglyi, S. I. and Golodets, V. Ya., Topological properties of full groups of measure space transformations, Sibirsk. Mat. Zh., 21 (1981), 3-14 (Russian); English transl. in Siberian Math. J.

[BG2] Groups of measure space transformations and invariants of outer conjugation for automorphisms from normalizers of type III full groups. J. Funct. Anal., 60 (1985). $341-369$.

[ChF] Chacon, R. V. and Friedman, N. A., Approximation and invariant measures, Z. Wahrscheinlichkeitstheorie Verw. Geb., 3 (1965), 286-295.

[C] Connes, A., Classification des factures, Proc. Symp. Pure Math., 38 (1982), 43-109. Amer. Math. Soc., Providence, RI.

[CK] Connes, A. and Krieger, W., Measure space automorphisms, the normalizers of their full groups, and approximate finiteness, J. Funct. Anal., 24 (1977), 336-352.

[Da] Danilenko, A. I., On topological structure of certain automorphism groups of an ergodic equivalence relation, Abstracts Amer. Math. Soc., 15 (1994), 403.

[D] Dobrovolski, T., Examples of topological groups homeomorphic of $l_{2}^{f}$, Proc. Amer. Math. Soc., 98 (1986), 303-311.

[FM] Feldman, J. and Moore, C. C., Ergodic equivalence relations, cohomology, and von Neumann algebras, I, Trans. Amer. Math. Soc., 234 (1977), 289-324.

[GeG] Gefter, S. L. and Golodets, V. Ya., Fundamental groups for ergodic actions and actions with unit fundamental groups, Publ. RIMS, Kyoto Univ., 24 (1988), 821-847.

[Ha] Halmos, P. R., Approximation theories for measure preserving transformations, Trans. Amer. Math. Soc., 55 (1944), 1-18.

[H] Hamachi, T., The normalizers group of an ergodic automorphism of type III and the commutant of an ergodic flow, J. Funct. Anal., 40 (1981), 387-403.

[HO] Hamachi, T. and Osikawa, M., Ergodic groups of automorphisims and Krieger's theorems, Sem. Math. Sci., Keio Univ., 3 (1981). 
[Har] Harada, S., Remarks on the topological group of measure preserving transformations, Proc. Japan Acad. 27 (1951), 523-526.

[IT] Ionesku Tulcea, A., On the category of certain classes of transformations in ergodic theory, Trans. Amer. Math. Soc., 114 (1965), 261-279.

[K] Keane, M., Contractibility of the automorphism group of a nonatomic measure space, Proc. Amer. Math. Soc., 26 (1970), 420-422.

[Krl] Krieger, W., On nonsingular transformations of a measure space. I, Z. Wahrscheinlichkeitstheorie Verw. Geb., 11 (1969), 83-97.

[Kr2] . On ergodic flows and isomorphisms of factors, Math. Ann., 223 (1976), 1970.

[M] Moore, C. C., Ergodic theory and von Neumann algebras, Proc. Symp. Pure Math., 38 (1982), 179-226. Amer. Math. Soc., Providence, RI.

[N] Nhu, N. T., The group of measure preserving transformations of the unit interval is an absolute retract, Proc. Amer. Math. Soc., 110 (1990), 515-522.

[PT] Popa, S. and Takesaki, M., The topological structure of the unitary and automorphism groups of a factor, preprint.

[R] Ramsay, A., The Mackey-Glimm dichotomy for foliations and other Polish groupoids, $J$. Funct. Anal., 94 (1990), 358-374.

[S1] Schmidt, K., Lectures on cocycles of ergodic transformation groups, Math. Inst. Univ. of Warwick, Coventry, 1976.

[S2] Algebraic ideas in ergodic theory, CBMS Regional Conf. Ser. in Math, Amer. Math. Soc., Providence, RI, 1990. 